\title{
Predictive monitoring for abnormal situation management
}

\author{
Ben C. Juricek ${ }^{a}$, Dale E. Seborg ${ }^{\mathrm{a}, *}$, Wallace E. Larimore ${ }^{\mathrm{b}}$ \\ ${ }^{a}$ Department of Chemical Engineering, University of California, Santa Barbara, CA 93106, USA \\ b Adaptics, Inc., 1717 Briar Ridge Rd., McLean, VA 22101, USA
}

\begin{abstract}
A novel process monitoring method is proposed that uses predictions from a dynamic model to predict whether process variables will violate an emergency limit in the future. The predictions are based on a Kalman filter and disturbance estimation. A critical feature of the proposed method is the evaluation of a $T^{2}$ statistic as a "reality check" for deciding if the future predictions are reliable and thus can be used for making control decisions. Several simulation examples demonstrate the effectiveness of the proposed technique for both linear and nonlinear processes, and for a variety of disturbances. (C) 2001 Elsevier Science Ltd. All rights reserved.
\end{abstract}

Keywords: Process monitoring; Prediction; Emergency limits

\section{Introduction}

The current status of an industrial process can be classified as one of three possible operating modes: normal, abnormal and emergency [1]. Statistically-based process monitoring techniques can detect when the plant state becomes "abnormal" by comparing current measurements with pre-determined limits. Such "alarm" limits can be calculated from the residuals of a stochastic process model, based on statistical descriptions of historical process data, or specified as acceptable bounds on process variability. A second set of wider limits are used to define "emergency" conditions. These conditions correspond to physical process limitations (e.g. the point that a pressure relief valve fails, a reaction runs away, etc.) or other limiting conditions that determine when a plant must be shutdown. Using the terminology in Nimmo [1], such a process limit is referred to in this paper as an "emergency limit".

By definition, an emergency limit violation is a very serious matter that can have catastrophic consequences. Such an "incident" can have a major economic impact on plant profitability due to loss of production or environmental sanctions. Thus there are significant incentives to develop reliable techniques that are capable of signaling future emergency limit violations early enough so that effective corrective action can be taken. In this

\footnotetext{
* Corresponding author.

E-mail addresses: juricek@engineering.ucsb.edu (B.C. Juricek), seborg@engineering.ucsb.edu (D.E. Seborg), larimore@adaptics.com (W.E. Larimore).
}

paper, a method is proposed that signals impending emergency limit violations by using predictions (or forecasts) from a process model. If an impending violation can be predicted with a sufficient warning period, then an effective corrective action may be able to prevent the predicted incident from actually occurring.

In this paper, the future predictions are initiated after a fault or abnormal situation has been detected. For example, the predictions could be calculated after an alarm limit violation occurs. This prediction step complements the more traditional fault diagnosis task that normally occurs after a fault has been detected. The proposed method predicts the future state of the process in the presence of unknown disturbances or faults, whereas traditional fault diagnosis methods are usually restricted to a set of faults that is specified a priori. Thus forecasting determines the effect of the fault on the process to better evaluate the severity of the situation; traditional diagnosis provides more precise information about specific faults.

The future forecasts can predict an impending emergency limit violation provided that the method for predicting and interpreting the future forecasts is reliable. Thus an objective of this research is to predict future outputs when there are large, sustained disturbances such as a ramp or a step change. Sustained process disturbances are particularly important because they are one of the main reasons why emergency limit violations occur. Thus the future predictions must be modified to account for the sustained disturbance appropriately. Two important issues for the proposed method are interpreting the future predictions and evaluating their reliability. 
The traditional approach for monitoring processes has been to perform limit checks on measurements and make simple engineering calculations (e.g. furnace efficiencies, mass balance closures). During the 1980s, the renewed emphasis on product quality and the successful experience in Japan, led to the widespread use of statistical quality control (SQC) methods and quality control charts such as Shewhart and CUSUM charts [2]. In recent years the development of improved techniques for on-line monitoring and fault detection has rapidly become a major research area, both in process control and in the more general control engineering field [3-7]. If an accurate dynamic model of the process is available, then effective fault detection and diagnosis strategies can be developed based on residual analysis and state and parameter estimation. The dynamic models can be either physical or empirical but most of the theoretical development has been for linear, discrete-time statespace models.

The research literature is replete with fault detection and diagnosis techniques based on linear process models [8-14]. However, the use of future forecasts to predict emergency limit violations has received little attention [15]. Nomikos and MacGregor [16] developed a technique for monitoring batch processes using forecasts of future variables and Multiway Principal Component Analysis (PCA). Chen and McAvoy [17] describe an analogous technique for continuous processes. Both techniques detect statistical anomalies rather than predict future emergency limit exceptions. In particular, forecasts of process variables are made assuming that future behavior will reflect the historical data that was used to build the PCA model.

A related research topic is the role that model predictions play in model predictive control, especially predictive control with state estimation $[18,19]$. In fact, the subject of the present paper could be considered "predictive monitoring". However, traditional MPC [20] has devoted surprisingly little attention to assessing the accuracy of the model predictions.

In the next section, the prediction of future outputs of a linear process in the presence of stochastic and unknown, deterministic disturbances is considered. The proposed method for using the future forecasts and assessing their reliability is then presented. Then the proposed methodology is demonstrated by a simulation study.

\section{Forecasts based on a dynamic model}

The theory for predicting future outputs from a discrete, dynamic, linear model is well known and closely related to the state estimation literature. The book by Goodwin and Sin [21] and the classical introduction by Gelb [22] are excellent references. Lütkepohl [23] provides a more statistically oriented reference for forecasting multivariable time series. The relevant prediction theory will be presented in this section.

The primary assumption for standard prediction methods is that the process of interest can be accurately described by a linear stochastic state space model,

$$
\begin{aligned}
x(k+1) & =A x(k)+B u(k)+D d(k)+w(k) \\
y(k) & =C x(k)+v(k)
\end{aligned}
$$

where $x$ is the vector of state variables $(n \times 1), u$ is the vector of measured inputs $(m \times 1), d$ is the vector of unmeasured disturbances $(r \times 1)$, and $y$ is the vector of measured outputs $(p \times 1)$. The process and measurement noise are denoted by $w$ and $v$. They are assumed to be uncorrelated with one another and described by zero mean, normal random vectors with covariance matrices, $Q$ and $R$.

The starting point for making future predictions is a steady-state Kalman filter [8,22] designed for stochastic disturbances only (i.e. assuming $d(k)=0$ ),

$$
\begin{aligned}
\hat{x}(k+1) & =\hat{A} x(k)+B u(k)+A K \epsilon(k) \\
\epsilon(k) & =y(k)-C \hat{x}(k) \\
K & =\bar{P} C^{T} \bar{\Sigma}^{-1}
\end{aligned}
$$

where $\bar{P}$ and $\bar{\Sigma}$ are the covariance matrices of the steady-state state estimate and measurement error. $\bar{P}$ satisfies the algebraic Riccati equation,

$\bar{P}-A \bar{P} A^{T}+A \bar{P} C^{T} \bar{\Sigma}^{-1} C \bar{P} A^{T}-Q=0$

and $\bar{\Sigma}$ is given by,

$\bar{\Sigma}=C \bar{P} C^{T}+R$

The state vector $x$ is assumed to be observable.

Equation (2) can be used to predict future values of $y$ if the future inputs are known and future stochastic disturbances are set equal to their expected values of zero. That is, the $N$-step ahead prediction can be calculated iteratively from (2). If $d(k)=0$, the future predictions will be unbiased with a covariance matrix determined by $Q, R$ and the covariance matrix propagation equations:

$$
\begin{aligned}
& P(k+N)=A P(k+N-1) A^{T}+Q \\
& \Sigma(k+N)=C P(k+N) C^{T}+R
\end{aligned}
$$

$P(k+N)$ is the covariance matrix of the N-step ahead prediction of the state estimation error, $\hat{x}(k+N)=$ $x(k+N)-\hat{x}(k+N \mid k)$ where $\hat{x}(k+N \mid k)$ is the prediction of the state at time $k+N$ using the information available at time $k . \Sigma(k+N)$ is the covariance matrix of the $N$-step ahead measurement prediction error, $\epsilon(k+N)$, conditional upon the measurements up to time $k$ : 


$$
\begin{aligned}
P(k+N) & =E\left[\hat{x}(k+N) \hat{x}^{T}(k+N) \mid y(k)\right] \\
\Sigma(k+N) & =E\left[\epsilon(k+N) \epsilon^{T}(k+N) \mid y(k)\right]
\end{aligned}
$$

where $E[x]$ denotes the expected value of $x$. The initial value, $P(k)=\bar{P}$, is provided by the algebraic Riccati Eq. (3).

\subsection{Predictions in the presence of deterministic disturbances}

If $d(k) \neq 0$, the disturbance must be accounted for in order to make accurate future predictions. This problem is closely related to accounting for nonrandom bias in estimation [24-26] A standard method for accommodating nonrandom bias in a Kalman filter is to augment the state vector with additional elements that correspond to the unknown biases. Although this approach is viable, the method described below results in the optimal least squares estimate for additive disturbances in linear systems and does not require tuning the filter using "fictitious noise" elements.

For the proposed application of using multi-step predictions for process monitoring, estimating the actual disturbances is less important than predicting future measurements accurately. If the $D$ matrix in (1) is known, the disturbance, $d(k)$, can be estimated and the term $D \hat{d}(k)$ added to (2). If $D$ is unknown, the effect of the term $D d(k)$ can be represented by,

$\delta(k)=D d(k)$

where the "pseudo-disturbance", $\delta(k)$, compensates for the unknown disturbance, $d(k)$. This term has no physical interpretation; it is used to update (2) in order to make accurate future predictions. The following approach is used to estimate this pseudo-disturbance:

1. Assume a particular form for the pseudo-disturbance (e.g. $\delta(k)$ is a step or a ramp).

2 . Estimate the parameters of $\delta(k)$ via least squares.

Clearly, a key issue in the proposed strategy is the assumed form of the pseudo-disturbance. The form must be chosen to balance accurately describing the actual phenomenon with the number of parameters to be estimated. The prediction method is best suited for sustained disturbances that can cause emergency violations and are easily predictable. Ramp and step disturbances are the simplest forms, if predictability is the goal. Of course, less accurate predictions will occur for other types of time-varying or nonstationary disturbances such as a random walk disturbance. But such disturbances are less likely to cause emergency limit violations. Again, the objective of the proposed prediction method is to predict emergency limit violations accurately, rather than provide accurate future predictions for all possible types of conditions.
The pseudo-disturbance parameters can be estimated from the Kalman filter residuals, $\epsilon(k)$. The residual can be written as,

$$
\epsilon(k)=\epsilon^{0}(k)+\rho\left(k, k_{0}\right)
$$

where $\epsilon^{0}(k)$ is the random part of the Kalman filter residual. If the specified covariance matrices $Q$ and $R$ are correct, the sequence $\epsilon^{0}$ is uncorrelated, normally distributed with zero mean and covariance $\bar{\Sigma}$ from (4). Basseville and Nikiforov refer to $\rho\left(k, k_{0}\right)$ as the "fault signature on the innovation" [8]; it represents the portion of the residual, $\epsilon(k)$, that is due to the nonrandom disturbance beginning at time $k_{0}$. It can be written as,

$\rho\left(k, k_{0}\right)=\sum_{i=k_{0}}^{k-1} C \bar{A}^{k-i-1} \delta(i)$

where

$\bar{A} \triangleq A(I-K C)$

For a particular form of $\delta(k)$, the fault signature can be written as the product of a known, time dependent matrix and a vector of unknown, constant parameters, $\delta$ :

$\rho\left(k, k_{0}\right)=\tilde{\rho}\left(k, k_{0}\right) \delta$

As is shown in the Appendix, the least squares solution for the parameters of $\delta$ can be written as,

$\hat{\delta}=\left[\sum_{i=k_{0}}^{k} \tilde{\rho}^{T}\left(i, k_{0}\right) \bar{\Sigma}^{-1} \tilde{\rho}\left(i, k_{0}\right)\right]^{-1}\left[\sum_{i=k_{0}}^{k} \tilde{\rho}^{T}\left(i, k_{0}\right) \bar{\Sigma}^{-1} \epsilon(i)\right]$

Example 1. Consider a two dimensional system with sampling period, $T_{\mathrm{s}}$. If $d(k)$ is assumed to be a ramp disturbance, then $\delta(k)$ can be written as,

$$
\begin{aligned}
\delta(k) & =\left[\begin{array}{cccc}
1 & 0 & T_{\mathrm{s}}\left(k-k_{0}\right) & 0 \\
0 & 1 & 0 & T_{\mathrm{s}}\left(k-k_{0}\right)
\end{array}\right]\left[\begin{array}{c}
\delta_{1,0} \\
\delta_{2,0} \\
\delta_{1, \mathrm{~s}} \\
\delta_{2, \mathrm{~s}}
\end{array}\right] \\
\delta(k) & =\Gamma\left(k, k_{0}\right) \delta \\
\Gamma\left(k, k_{0}\right) & \triangleq\left[\begin{array}{cccc}
1 & 0 & T_{\mathrm{s}}\left(k-k_{0}\right) & 0 \\
0 & 1 & 0 & T_{\mathrm{s}}\left(k-k_{0}\right)
\end{array}\right] \\
\delta & =\left[\begin{array}{llll}
\delta_{1,0} & \delta_{2,0} & \delta_{1, \mathrm{~s}} & \delta_{2, \mathrm{~s}}
\end{array}\right]^{T}
\end{aligned}
$$

The pseudo-disturbance assumes that two distinct ramp disturbances occur, one for each state variable in Eq. (1). The intercepts of the ramps for the start time, $k_{0}$, are given by $\left(\delta_{1,0}, \delta_{2,0}\right)$ and the slope of the ramps are 
given by $\left(\delta_{1, \mathrm{~s}}, \delta_{2, \mathrm{~s}}\right)$. The known term of the fault signature on the innovation becomes,

$\tilde{\rho}\left(k, k_{0}\right)=\sum_{i=k_{0}}^{k-1} C \bar{A}^{k-i-1} \Gamma\left(i, k_{0}\right)$

Eq. (11) can be used to compute $\hat{\delta}$.

The covariance of $\hat{\delta}, \Sigma_{\hat{\delta}, \hat{\delta}}$, and the cross-covariance between $\hat{x}$ and $\hat{\delta}, \Sigma_{\hat{\delta}, \hat{x}}$, are given by,

$\Sigma_{\hat{\delta}, \hat{\delta}}=\left[\sum_{i=k_{0}}^{k} \tilde{\rho}^{T}\left(i, k_{0}\right) \bar{\Sigma}^{-1} \tilde{\rho}\left(i, k_{0}\right)\right]^{-1}$

$\Sigma_{\hat{\delta}, \hat{x}}=\left[\sum_{i=k_{0}}^{k} \tilde{\rho}^{T}\left(i, k_{0}\right) \bar{\Sigma}^{-1} \tilde{\rho}\left(i, k_{0}\right)\right]^{-1}\left[\sum_{i=k_{0}}^{k} \tilde{\rho}^{T}\left(i, k_{0}\right) \bar{\Sigma} C P(i)\right]$

The Kalman filter equations in (2) are modified by including $\hat{\delta}(k)=\Gamma(k) \hat{\delta}$.

$$
\begin{aligned}
\hat{x}^{m}(k+1) & =A \hat{x}^{m}(k)+B u(k)+\hat{\delta}(k)+A K \epsilon^{m}(k) \\
y(k) & =C \hat{x}^{m}(k)+\epsilon^{m}(k)
\end{aligned}
$$

The superscript $m$ differentiates the modified Kalman filter Eq. (15) from the standard Kalman filter in (2).

Future predictions are made by assuming that the pseudo-disturbance remains constant for the prediction horizon and then setting $\epsilon^{m}(k)$ equal to zero for all future values:

$$
\begin{aligned}
\hat{y}(k+N)= & C A^{N} \hat{x}^{m}(k)+\sum_{i=0}^{N-1} C A^{N-i-1} B u(k+i) \\
& +\sum_{i=0}^{N-1} C A^{N-i-1} \hat{\delta}(k+i) \\
= & C A^{N} \hat{x}^{m}(k)+\sum_{i=0}^{N-1} C A^{N-i-1} B u(k+i) \\
& +\sum_{i=0}^{N-1} C A^{N-i-1} \Gamma(k+i) \hat{\delta}
\end{aligned}
$$

The corresponding equations for the covariance matrices in (5) must be modified to include $\Sigma_{\hat{\delta}, \hat{\delta}}$ and $\Sigma_{\hat{\delta}, \hat{x}}$ to account for the uncertainty in estimating the pseudodisturbance,

$$
\begin{aligned}
P^{m}(k+N)= & A P^{m}(k+N-1) A^{T}+Q \\
& +\Gamma(k+N-1) \Sigma_{\hat{\delta}, \hat{\delta}} \Gamma(k+N-1)^{T} \\
& +A \Sigma_{\hat{\delta}, \hat{x}}^{T} \Gamma(k+N-1)^{T} \\
& +\Gamma(k+N-1) \Sigma_{\hat{\delta}, \hat{x}} A^{T} \\
\Sigma^{m}(k+N)= & C P^{m}(k+N) C^{T}+R
\end{aligned}
$$

where $\Sigma^{m}$ and $P^{m}$ denote the covariance matrices for the predictions of the output and state variables using the modified Kalman filter.

The regressors in (11), $\tilde{\rho}\left(k, k_{0}\right)$, can be precomputed for a given start time and disturbance form. Thus, the prediction calculations are suitable for on-line implementation.

As mentioned earlier, the approach suggested in most of the standard filtering books [22] for estimating unknown non-deterministic disturbances involves augmenting the original state vector, $x$, with additional state variables corresponding to $d(k)$ or the pseudo-disturbance model parameters. A preliminary investigation indicated that the predicted outputs were very sensitive to the design of the Kalman filter. Specifically, estimating an augmented state requires specifying the variance information of a "fictitious noise"; i.e. the elements of $Q$ that include the augmented state variable. In reality, these elements are zero because the disturbance is not random. However, the augmented Kalman filter does not update these state variables unless the fictitious variance values are nonzero. Thus, the accuracy of the estimated parameters is quite sensitive to the magnitudes of these specified values. Due to the recursive relation in (17), the increase in the variance of future forecasts also depends strongly on the assumed fictitious noise variance. Because the least squares approach described above is simpler and more accurate, it was adopted.

\section{Early detection of an emergency situation}

In the proposed approach, the future predictions from the modified Kalman filter are used to determine whether the process will violate an emergency limit. The covariance information in (17) can be used in conjunction with the future redictions to determine the probability that an emergency limit will be violated. The predictions are based on several assumptions: an accurate linear model, known noise statistics, correct disturbance form, etc. Before taking any corrective action based on the future predictions, the performance of past predictions should be investigated. This "reality check" verifies that the assumptions are valid.

If all of the assumptions are correct, the residuals of (16) are distributed as a multivariate normal vector, with zero mean and an estimated covariance matrix given by (17). Therefore, the $i$ th residual will have a $t$-distribution with sample variance given by the $i$ th diagonal element of $\Sigma^{m}$. Hence, $100(1-\alpha) \%$ confidence limits of the $N$ step ahead prediction for $y_{i}$ can be constructed from,

$y_{i}(k+N)=\hat{y}_{i}(k+N / k) \pm t_{n-q-p+1}(\alpha) \Sigma_{i, i}^{m}(k+N)^{\frac{1}{2}}$

where $n$ is the number of data points used to estimate the pseudo-disturbance, $p$ is the dimension of $y, q$ is the 
number of parameters estimated in the regression, and $t_{n-q-p+1}(\alpha)$ is the critical value from the $t$-distribution with $n-q-p+1$ degrees of freedom. If both the upper and lower limits of a future prediction exceed the emergency limit, we conclude that a future violation will occur at the $(1-\alpha) 100 \%$ confidence level.

But due to such factors as model mismatch, process nonlinearities, and disturbances that cannot be accurately described by the assumed disturbance form, the theoretical covariance of future forecast errors in (17) may over-estimate the actual accuracy of the predictions. In order to evaluate the actual prediction accuracy, a $T^{2}$ statistic [27] is calculated based on the performance of previous predictions. The $T^{2}$ statistic is a general multivariate statistic for determining if the actual residual vector is zero mean, assuming that the estimated covariance matrix is correct. For example, at time $k^{*}=k+N$, the accuracy of the predictions made at time $k$ can be checked via the statistic,

$$
\begin{aligned}
T^{2}\left(k^{*}, k\right) & =\epsilon^{m}\left(k^{*} \mid k\right)^{T} \Sigma^{m}\left(k^{*}\right)^{-1} \epsilon^{m}\left(k^{*} \mid k\right) \\
\epsilon^{m}\left(k^{*} \mid k\right) & =y\left(k^{*}\right)-\hat{y}\left(k^{*} \mid k\right)
\end{aligned}
$$

The $T^{2}$ statistic can be compared to the following $100(1-\alpha) \%$ limit [27]:

$T_{p, n-q}^{2}(\alpha)=\frac{p(n-q)}{n-p-q+1} F_{p, n-q-p+1}(\alpha)$

This limit reflects the uncertainty of estimating the $q$ parameters of $\delta$ with $n$ data points for a $p$-dimensional measurement vector.

The $T^{2}$ statistic provides a useful measure of the prediction accuracy of the past forecasts. Hence, the validity of the required assumptions and the reliability of current predictions can be assessed based on the $T^{2}$ statistic. For example, suppose that the current predictions and confidence limits signal an impending emergency situation, but the $T^{2}$ values from (19) exceed the limit in (20) indicating that the past predictions are incorrect. Then the signal for a predicted violation should be ignored, because the linear model, noise statistics, or disturbance form is not correct, and thus the current predictions are not reliable. The $T^{2}$ statistic is qualitatively similar to other statistical tests on future forecasts described by Lütkepohl [23].

The proposed procedure can be summarized by the following steps:

1. Begin the analysis. This could be done on demand or triggered by a fault detection method, as described below.

2. Estimate a "pseudo-disturbance", $\delta(k)$, to compensate for deterministic disturbances and plant-model mismatch.

3. Predict future values of $y$ using the modified Kalman filter in (16).
4. Calculate the corresponding covariance matrices for the predictions using (17).

5. Construct confidence limits for the predictions using (18).

6. Determine the accuracy of the past predictions of the current measurement by calculating the $T^{2}$ statistic in (19).

7. If both confidence bounds violate an emergency limit and $T^{2}\left(k^{*}, k\right)<T_{p, n-q}^{2}(\alpha)$, an early warning of an emergency situation occurs; otherwise, the future predictions are ignored at the current time.

The proposed approach requires specifying a confidence level, $1-\alpha$, prediction horizon, $N_{\mathrm{p}}$, and form of $\delta(k)$ (i.e. step, ramp, etc.). The most important decision is the pseudo-disturbance form.

The predictions can be initiated once a fault or disturbance is detected, an alarm limit is violated, or simply on user demand. Additive disturbances can be detected by several methods, including a $\chi^{2}$ test on the Kalman filter residuals. The start time, $k_{0}$, also can be determined in a number of ways. Because the proposed forecasting method assumes that a process model is available and the statistical properties of $v(k)$ and $w(k)$ are known, a generalized likelihood ratio (GLR) test [8] can be used to determine the time that the disturbance began. However, the precise start time is not as important for predictions as simply detecting that a disturbance has occurred. The fault detection and start time determination issues are described in greater detail in $[8,11,28]$. For the simulation examples in the next section, $k_{0}$ is set equal to the true value of $k$ when the disturbance occurs.

\section{Simulation results}

Two simulation examples are used to demonstrate the proposed methodology: a hypothetical SISO process and a continuous stirred tank reactor (CSTR) with two inputs and two outputs.

\subsection{SISO example}

Consider a process model given by,

$y(k)=\frac{1}{q-0.95} w(k)+\frac{1}{q-0.95} d(k)+v(k)$

where $q$ is the forward shift operator, and $w(k)$ and $v(k)$ have zero means and variances of $Q=R=0.1$. The disturbance, $d(k)$, is a step change at time, $k=101$. The emergency limit is set at $y=20$. For the simulation, $d(k)=1.3$ for $k>100$, which causes a violation at $k=128$. The results are shown in Fig. 1. The lefthand portion of each figure shows the predictions made at time $k^{*}$, for 

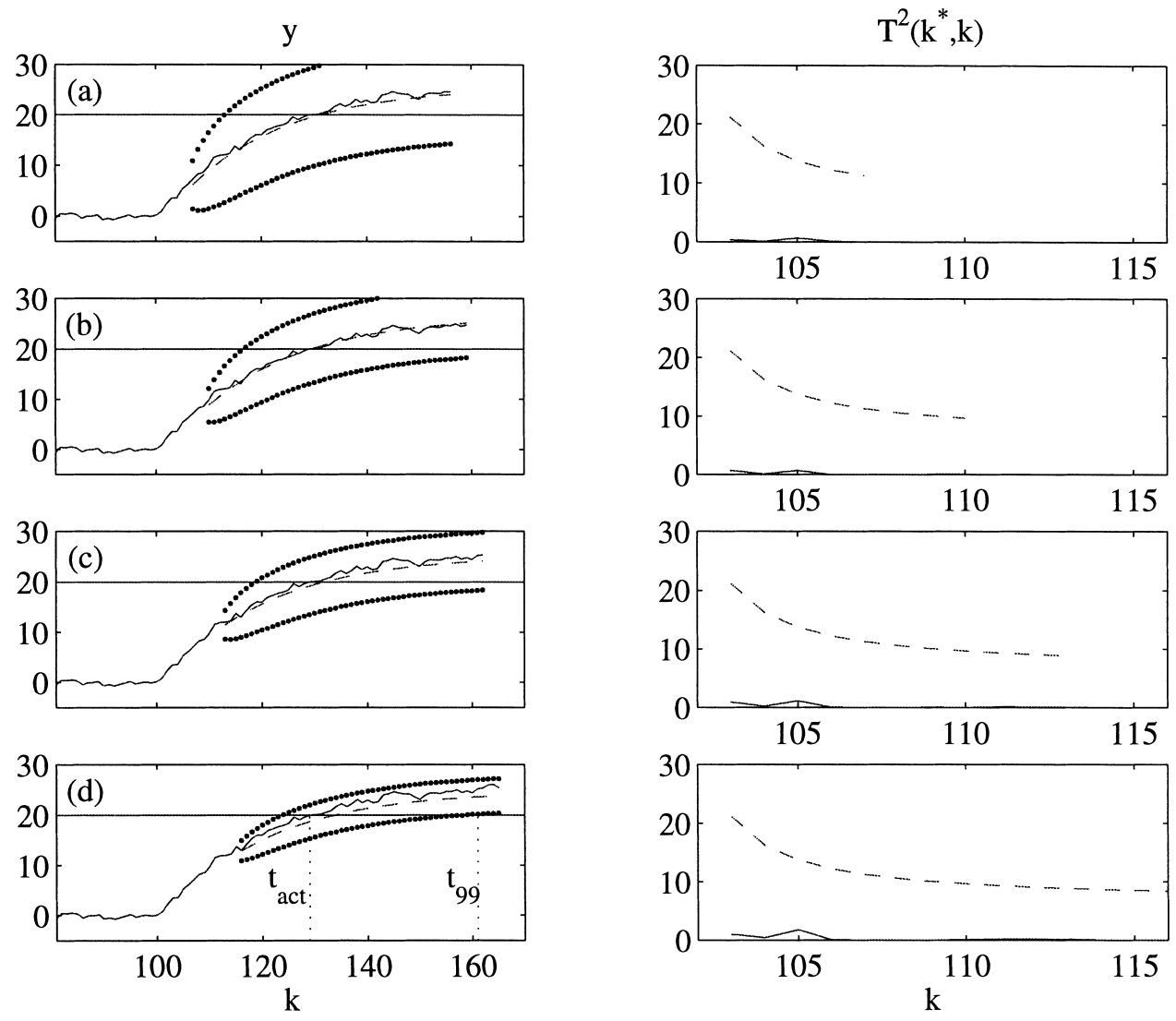

Fig. 1. SISO example, $d(k)=1.3$ : (a) $k^{*}=107$, (b) $k^{*}=110$, (c) $k^{*}=113$, (d) $k^{*}=116$.

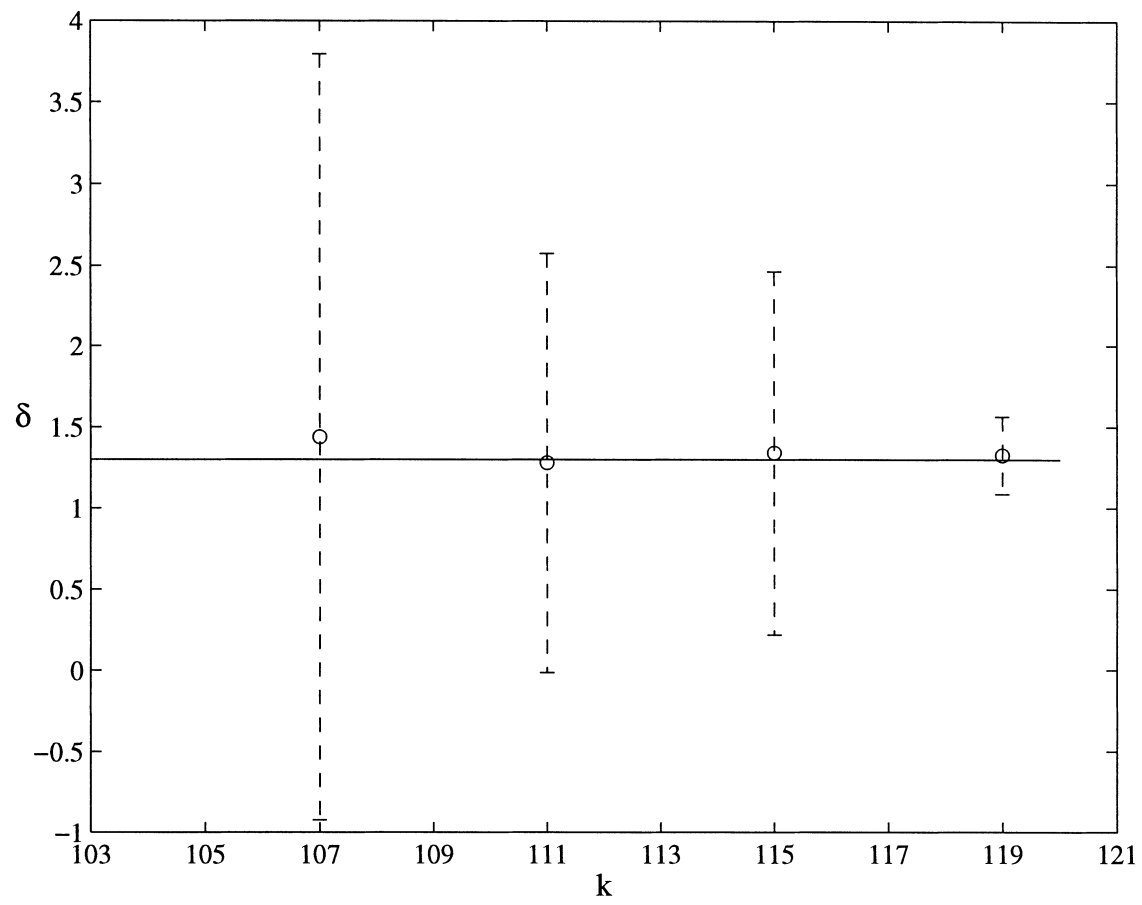

Fig. 2. The $95 \%$ errorbars for $\hat{\delta}$. 
different values of $k^{*}$. The dashed line represents the predicted future values, $\hat{y}\left(k+N \mid k^{*}\right)$, for $N=(1,2, \ldots$, $50)$. The dotted lines represent the $95 \%$ confidence limits for the future predictions. The solid line is the actual value of $y$. The calculated $T^{2}$ statistics for time $k^{*}$ are shown as the solid line in the righthand plots. $T^{2}\left(k^{*}, k\right)$ characterizes the accuracy of the predictions of $\hat{y}\left(k^{*} \mid k\right)$ where $k^{*}-N_{\mathrm{p}}<k<k^{*}$. The dashed line is the confidence limit from (20). It varies with $k$ because the number of data points used to calculate $\hat{y}\left(k^{*} / k\right)$ also varies with $k$.

For the predictions made at $k^{*}=116$, the lower confidence limit exceeds the emergency limit of $y=20$. The time that both confidence limits violate the emergency limit is shown by $t_{99}$ in Fig. 1. The actual time that the limit is exceeded is represented by $t_{\text {act }}$. Thus the warning period given by the prediction analysis, denoted by $\Delta t_{\mathrm{w}}$, is $t_{\text {act }}-k^{*}$. Because the warning period is the time available for taking corrective action, $\Delta t_{\mathrm{w}}$ is an important metric for the prediction analysis. The $T^{2}$ values for $k^{*}=116$ suggest that the future predictions can be believed, based on the prediction of $y(116)$ made earlier. Thus, a limit violation is predicted. Because the actual violation occurs at $k=128, \Delta t_{\mathrm{w}}=128-116=12$ samples.

The estimates of $\delta$ made at time $k$ are shown in Fig. 2. In this case, $d(k)=\delta(k)=\delta$. The estimated values of $\hat{\delta}$ are denoted by the small circles and the $99 \%$ confidence limits are indicated by the dashed lines. The solid line denotes the true value of 1.3. The estimated value of $\delta$ quickly converges to the true value, and the true value is always within the confidence limits.
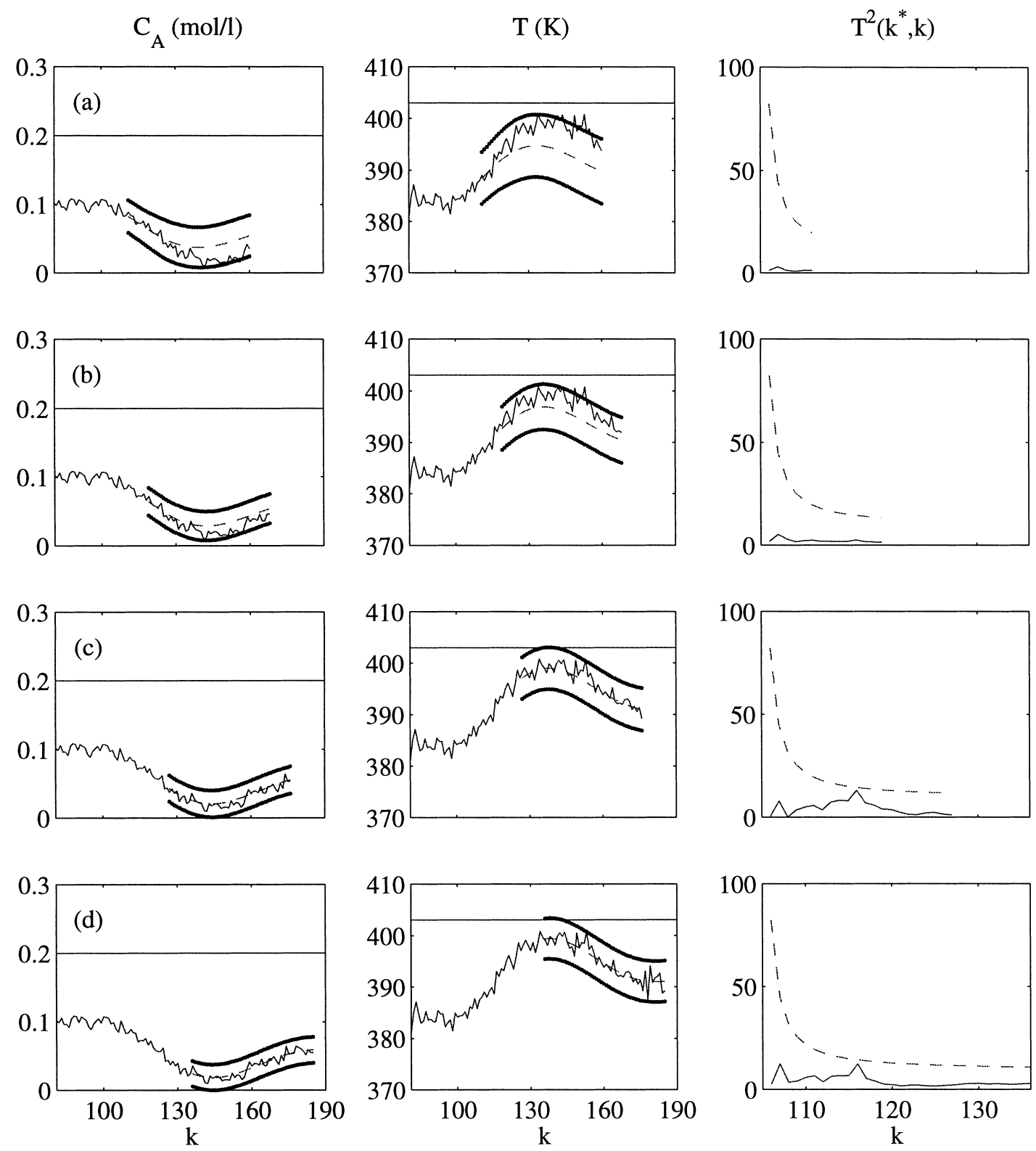

Fig. 3. $+20 \mathrm{~K}$ step in $T_{\mathrm{F}}$ that does not cause a future violation: (a) $k^{*}=113$, (b) $k^{*}=120$, (c) $k^{*}=127$, (d) $k^{*}=134$. 


\subsection{CSTR example}

A nonisothermal CSTR with a first-order, irreversible reaction $A \rightarrow B$, first-order kinetics, and a cooling jacket to remove heat, is described by [29]:

$\frac{\mathrm{d} C_{A}}{\mathrm{~d} t}=\frac{F}{V}\left(C_{\mathrm{F}}-C_{\mathrm{A}}\right)+k_{0} \exp \left(-\frac{E}{R T}\right) C_{\mathrm{A}}$

$$
\begin{aligned}
\frac{\mathrm{d} T}{\mathrm{~d} t}= & \frac{F}{V}\left(T_{\mathrm{F}}-T\right)+\frac{-\Delta H}{\rho C_{\mathrm{p}}} k_{0} \exp \left(-\frac{E}{R T}\right) C_{\mathrm{A}} \\
& +\frac{U A}{V \rho C_{\mathrm{p}}}\left(T_{\mathrm{J}}-T\right)
\end{aligned}
$$

The parameter values used in the simulations are shown in Table 1.
The nonlinear equations are discretized and linearized to provide a linear, discrete-time model with two outputs $\left(C_{\mathrm{A}}\right.$ and $\left.T\right)$, two inputs $\left(F\right.$ and $\left.T_{\mathrm{J}}\right)$, and two disturbances $\left(C_{\mathrm{F}}\right.$ and $\left.T_{\mathrm{F}}\right)$. For the following simulations, either $T_{\mathrm{F}}$ or $C_{\mathrm{F}}$ will act as the disturbance. Process and

Table 1

CSTR Parameters

\begin{tabular}{llll}
\hline Variable & Value & Variable & Value \\
\hline$F$ & $1001 / \mathrm{min}$ & $\mathrm{E} / \mathrm{R}$ & $8750 \mathrm{~K}$ \\
$C_{\mathrm{F}}$ & $1 \mathrm{~mol} / 1$ & $k_{0}$ & $7.2 \times 10^{10} / \mathrm{min}$ \\
$T_{\mathrm{F}}$ & $350 \mathrm{~K}$ & $U A$ & $5 \times 10^{4} \mathrm{~J} / \mathrm{min} \cdot \mathrm{K}$ \\
$V$ & 1001 & $T_{\mathrm{J}}$ & $309.9 \mathrm{~K}$ \\
$\rho$ & $1 \mathrm{~kg} / 1$ & $-\Delta H$ & $5 \times 10^{4} \mathrm{~J} / \mathrm{mol}$ \\
$C_{p}$ & $239 \mathrm{~J} / \mathrm{kg} \cdot \mathrm{K}$ & & \\
\hline
\end{tabular}

$\mathrm{T}(\mathrm{K})$

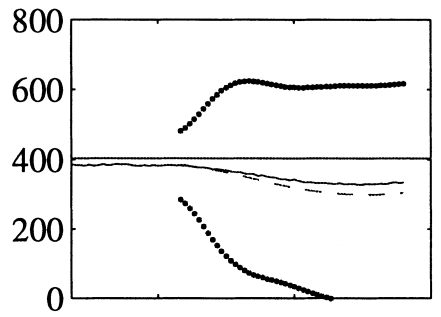

$\mathrm{T}^{2}\left(\mathrm{k}^{*}, \mathrm{k}\right)$
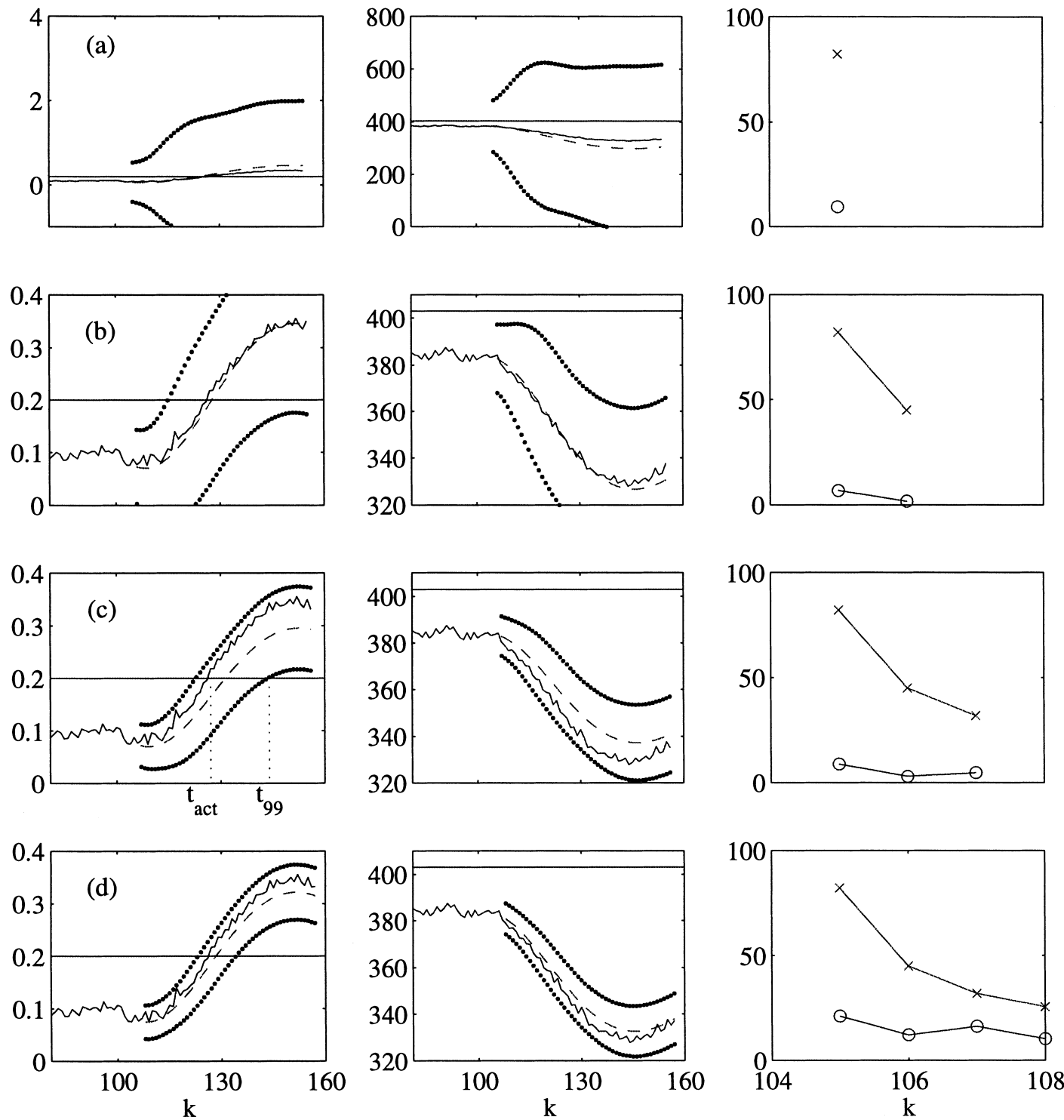

Fig. 4. $-0.375 \mathrm{~mol} / 1$ step in $C_{\mathrm{F}}$ : (a) $k^{*}=105$, (b) $k^{*}=106$, (c) $k^{*}=107$, (d) $k^{*}=108$. 
measurement noise are included in order to achieve a reasonable signal to noise ratio. The disturbance occurs at $k=101$ and forecasts of the next 50 measurements $\left(N_{\mathrm{p}}=50\right)$ are made beginning at $k^{*}=105$.

The results for a $20 \mathrm{~K}$ step disturbance in $T_{\mathrm{F}}$ are shown in Fig. 3. In this case, the assumed pseudo-disturbance form is also a step change beginning at $k=$ 101. The solid horizontal lines at $C_{\mathrm{A}}=0.2 \mathrm{~mol} / 1$ and $T=403 \mathrm{~K}$ indicate the emergency limits. The future predictions are shown by the dashed line, and the confidence limits for the forecasts are denoted by the dotted lines. The measured values are indicated by the solid lines. Although the predictions are made based on the data up to time $k^{*}$, the next 50 measurements are shown in order to compare the predictions with the actual future values. The plots of the $T^{2}\left(k^{*}, k\right)$ statistic are shown beside each set of predictions. The $99 \% T^{2}$ limit from (20) is indicated by the monotonically decreasing dashed line, and the $T^{2}$ statistic is indicated by the solid line.

The predictions for the step change in $T_{\mathrm{F}}$ are generally accurate. Neither the predictions nor the actual values violate the emergency limits. Because the $T^{2}$ statistic is always within statistical limits, the predictions are accepted as valid. The earliest predictions, made at $k^{*}=110$, are less accurate than those made later, due to
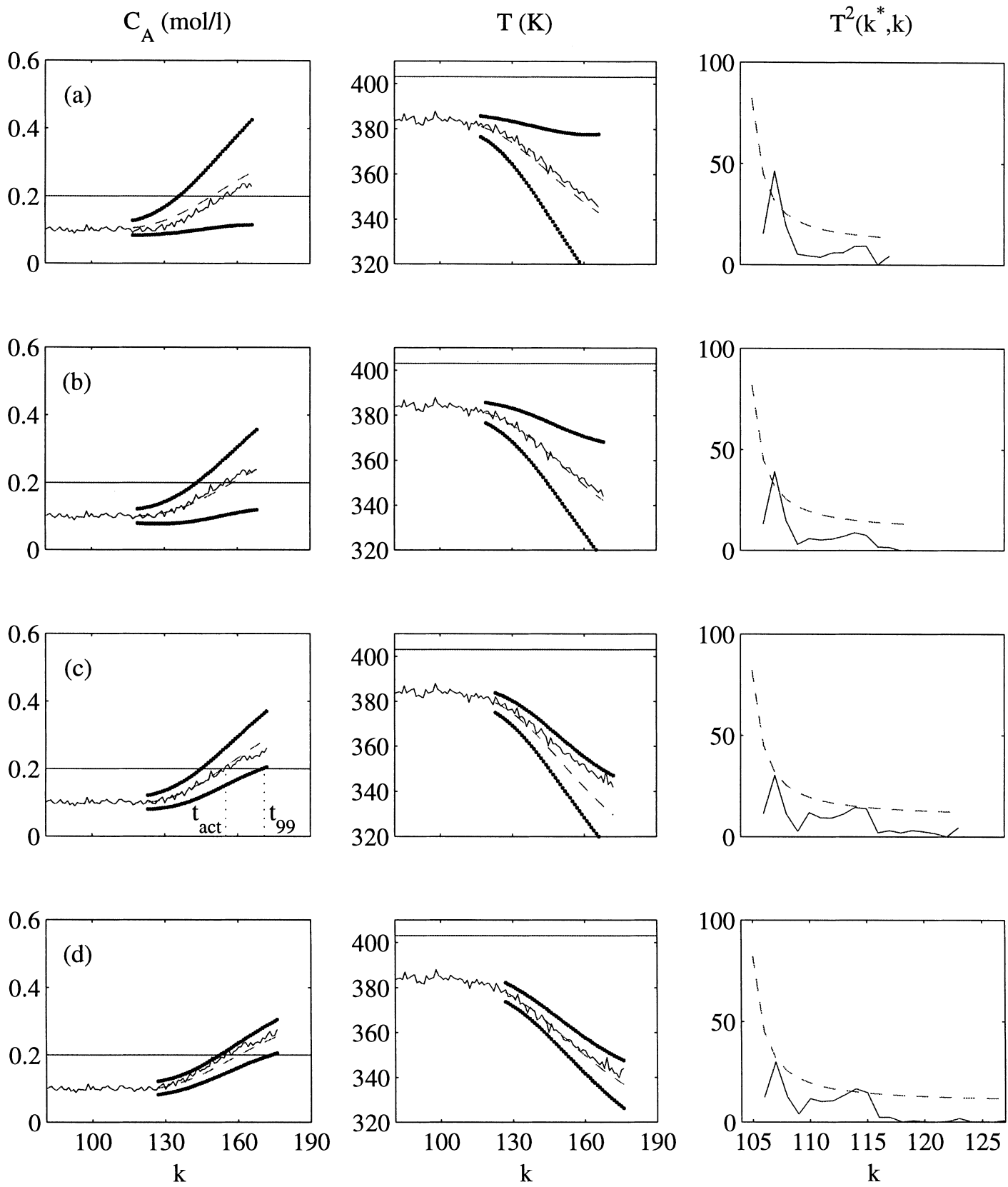

Fig. 5. $-0.006 \mathrm{~mol} / \mathrm{l} \cdot \mathrm{min}$ ramp disturbance in $C_{\mathrm{F}}$ : (a) $k^{*}=118$, (b) $k^{*}=120$, (c) $k^{*}=123$, (d) $k^{*}=127$. 
the small amount of data used to estimate the pseudodisturbance. As more data become available, the prediction accuracy improves. The prediction accuracy is reflected by the relatively wide confidence bounds after a few measurements, and the progressively narrowing bounds as more data become available.

The predictions for a $-0.375 \mathrm{~mol} / 1$ step change in $C_{\mathrm{F}}$ are shown in Fig. 4. In this case, the $C_{\mathrm{A}}$ measurement exceeds the emergency limit at $k=129$. As before, the assumed pseudo-disturbance form is also a step. The confidence limits at time $k^{*}=105$ and 106 are too wide to make any meaningful statements about the future process variables. But at $k^{*}=107$ the confidence limits are narrow, and both limits for the $C_{\mathrm{A}}$ predictions vio- late the emergency limit. Because the $T^{2}$ statistic values are below the theoretical limits, a prediction of an impending violation is signaled. Since the predictions are made at $k^{*}=107$ and the actual violation occurs at $k=129$, the warning period is $\Delta t_{\mathrm{w}}=22$. Hopefully this warning period would be sufficient to allow a corrective action to prevent the emergency limit violation.

The predictions for a $-0.006 \mathrm{~mol} / 1 \cdot \mathrm{min}$ ramp disturbance in $C_{\mathrm{F}}$ are shown in Fig. 5. The pseudo-disturbance form is also a ramp. Again, the confidence limits narrow after more data become available for estimating the pseudo-disturbance. At $k^{*}=117$, both confidence limits for $C_{\mathrm{A}}$ exceed the emergency limit. Because the $T^{2}$ statistics do not reject the forecasts of the current time
$\mathrm{C}_{\mathrm{A}}(\mathrm{mol} / \mathrm{l})$
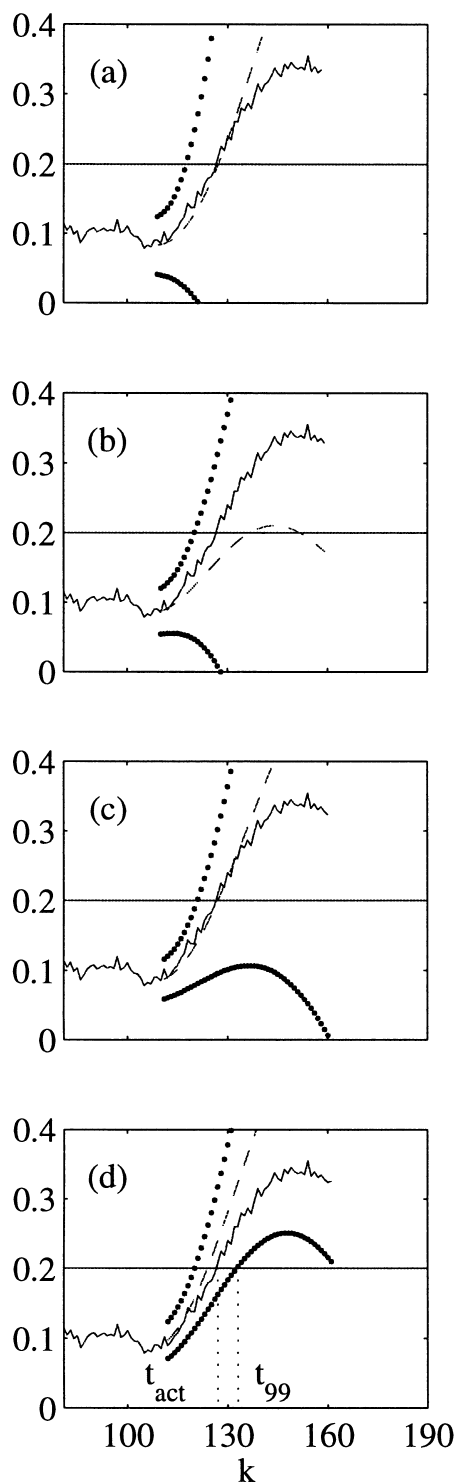

$\mathrm{T}(\mathrm{K})$
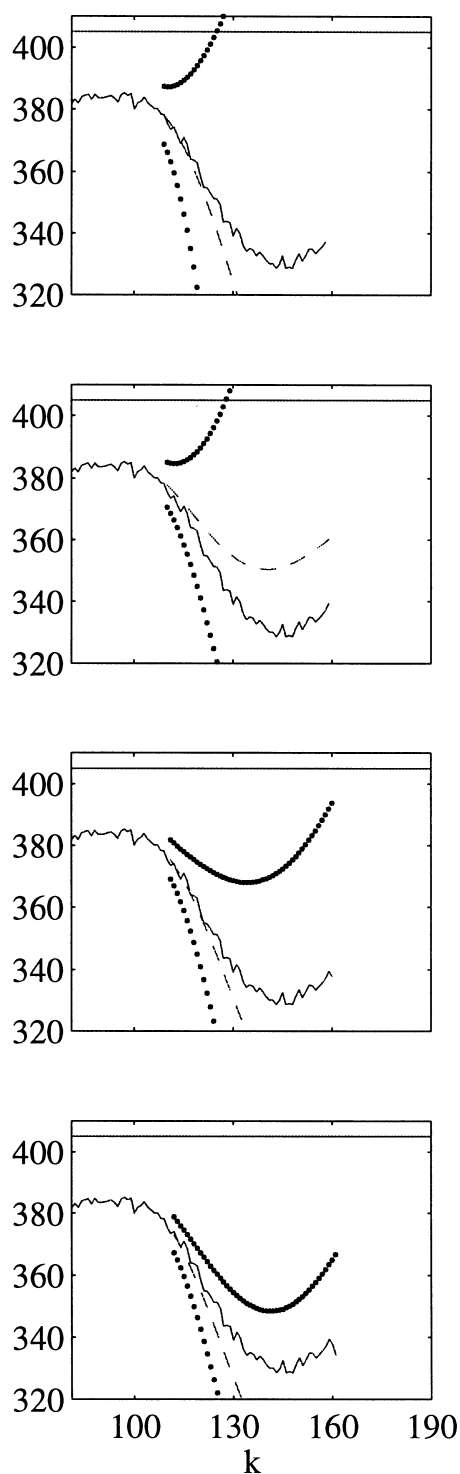

$\mathrm{T}^{2}\left(\mathrm{k}^{*}, \mathrm{k}\right)$
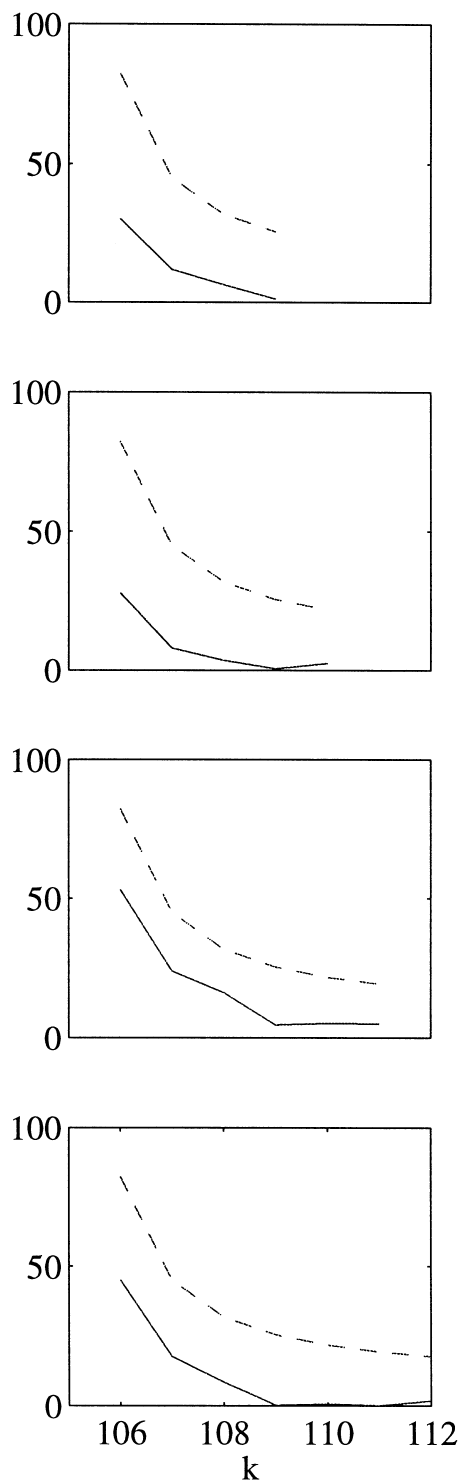

Fig. 6. $-0.375 \mathrm{~mol} / 1 \mathrm{step}$ in $C_{\mathrm{F}}$; a ramp disturbance form is used for estimating the pseudo-disturbance: (a) $k^{*}=109$, (b) $k^{*}=110$, (c) $k^{*}=111$, (d) $k^{*}=112$. 
that were made in the past, the future violation is signaled. The actual violation occurs at $k=144$ and thus $\Delta t_{\mathrm{w}}=27$.

The previous simulations were ideal because the assumed form for the pseudo-disturbance was correct. If a ramp disturbance form is assumed rather than a step, the future predictions should still be accurate for a step disturbance. The converse is not true; a step pseudo-disturbance cannot accurately predict future measurements when the true disturbance is a ramp. Fig. 6 shows the transient response to the same $-0.375 \mathrm{~mol} / 1$ step change in $C_{\mathrm{F}}$ as in Fig. 4, but now a ramp pseudo-disturbance is assumed. Due to the two extraneous disturbance parameters that must be estimated, the covariance matrix for the parameter estimates has more elements, leading to larger confidence limits for the future predictions. In this case, the future violation is predicted at $k^{*}=112$ and $\Delta t_{\mathrm{w}}=17$. A comparison of Figs. 4 and 6 indicates that the penalty for using the incorrect disturbance form is that $\Delta t_{\mathrm{w}}$ is reduced by five samples. The following simulations use a ramp form for the pseudo-disturbance.

Figure 7 shows simulated results for closed-loop operation and the $-0.375 \mathrm{~mol} / 1$ step disturbance in $C_{\mathrm{F}}$. Two PI controllers are used with gains, $K_{\mathrm{c}}\left(F, C_{\mathrm{A}}\right)=150$ $1^{2} / \mathrm{min} \cdot \mathrm{mol}$ and $K_{\mathrm{c}}\left(T_{\mathrm{J}}, T\right)=0.2$, and integral time constants, $\tau_{\mathrm{I}}\left(F, C_{\mathrm{A}}\right)=150 \mathrm{~min}$ and $\tau_{\mathrm{I}}\left(T_{\mathrm{J}}, T\right)=0.2 \mathrm{~min}$. Saturation limits of $\pm 101 / \mathrm{min}$ are placed on $F$ and \pm 10 $\mathrm{K}$ on $T_{\mathrm{J}}$. The future inputs are calculated from the control algorithm using the future predictions. After the
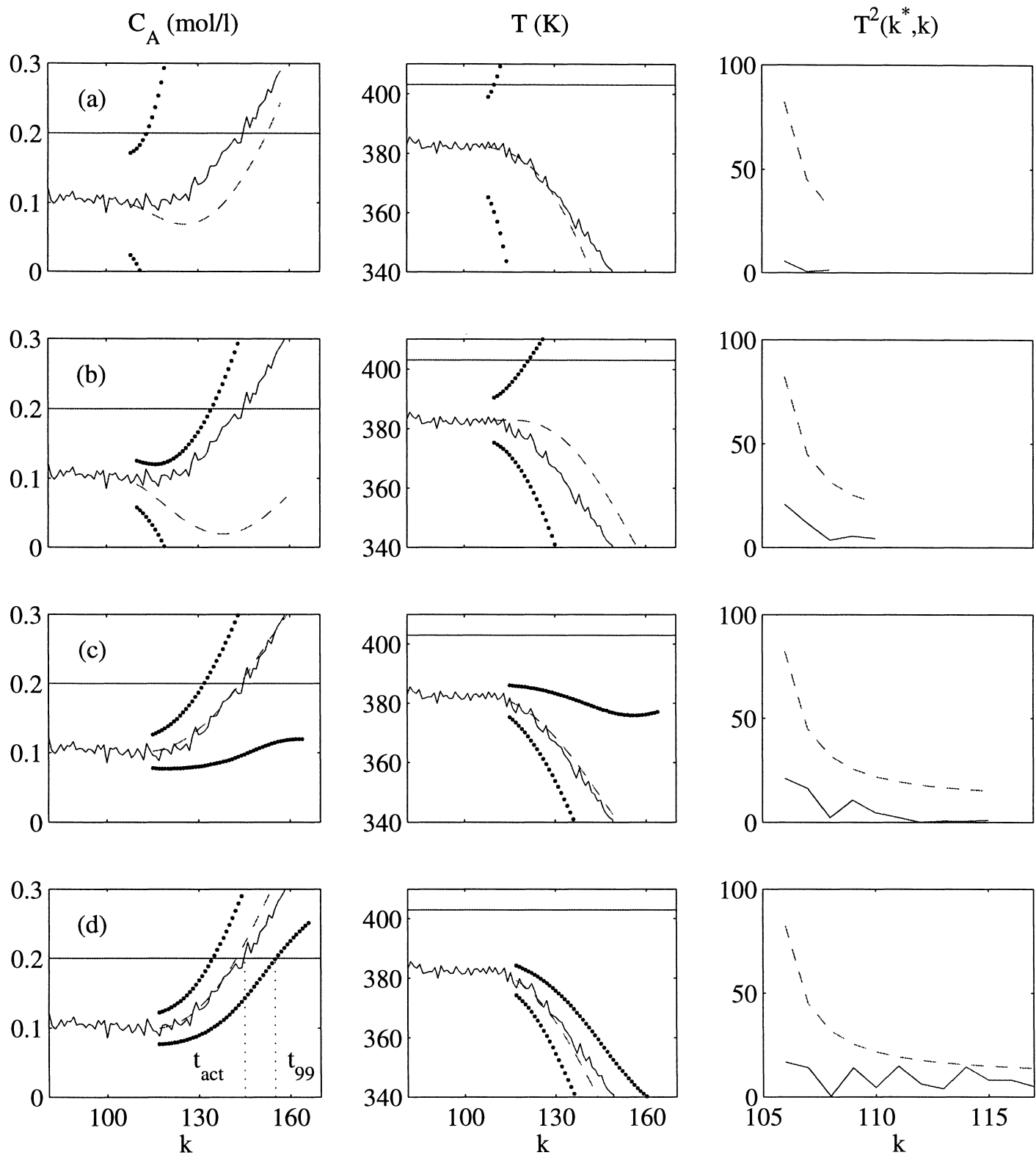

Fig. 7. $-0.375 \mathrm{~mol} / 1$ step in $C_{\mathrm{F}}$ and closed-loop operation: (a) $k^{*}=108$, (b) $k^{*}=110$, (c) $k^{*}=115$, (d) $k^{*}=117$. 
manipulated variables saturate, $C_{\mathrm{A}}$ exceeds its upper limit at $k=146$. Predictions during the closed-loop operation provide an early warning at $k^{*}=117$ and thus $\Delta t_{\mathrm{w}}=29$. By contrast, open-loop predictions for the same disturbance in Fig. 4 resulted in $\Delta t_{\mathrm{w}}=22$.

The following simulations examine three important situations where the proposed methodology can fail to accurately forecast future measurements: plant-model mismatch (parametric errors in the linear model), random walk disturbances, and process nonlinearities. In these situations, the $T^{2}$ statistic will be important for determining whether the predictions are reliable.
The effect of parametric errors is evaluated by simulating coolant jacket fouling. Because the process model is used for estimating the pseudo-disturbance in (11), a model error leads to biased estimates of $\delta$. The bias is unimportant, however, if the model can still accurately predict future measurements. In Fig. 8 a process model with a $10 \%$ smaller UA value is used when the -0.375 mol/1 step in $C_{\mathrm{F}}$ disturbance occurs during open-loop operation. The predictions based on the inaccurate model successfully predict the violation at $k^{*}=117$, and $\Delta t_{\mathrm{w}}=12$. Comparing this result with $\Delta t_{\mathrm{w}}$ for the correct process model in Fig. $6, \Delta t_{\mathrm{w}}$ decreases by five samples.

$$
\mathrm{C}_{\mathrm{A}}(\mathrm{mol} / \mathrm{l})
$$
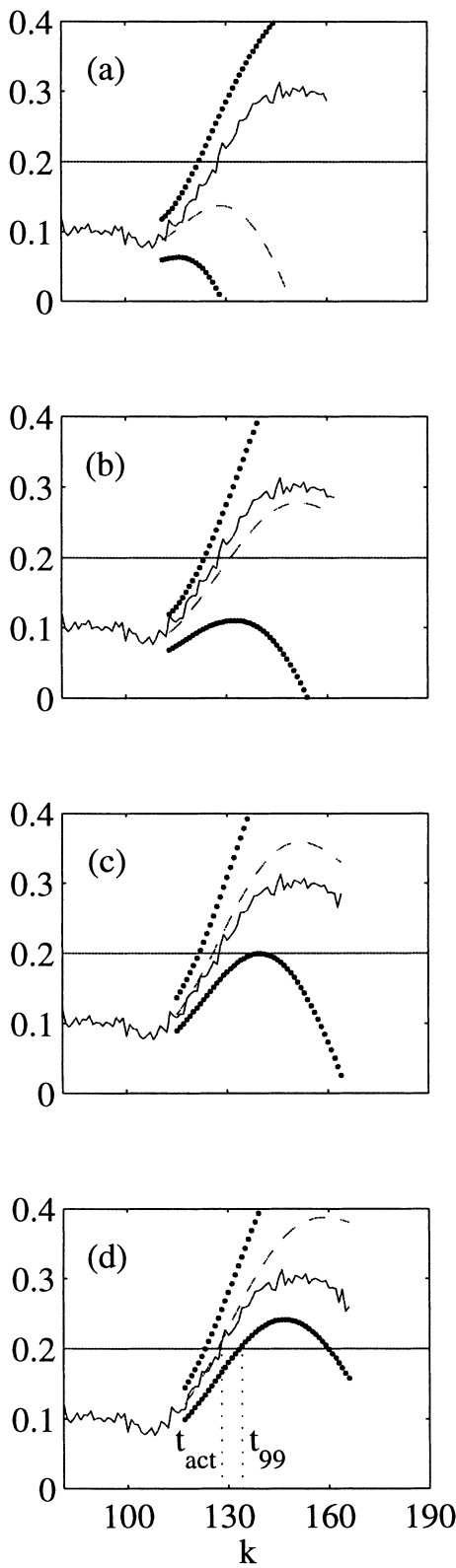

$\mathrm{T}(\mathrm{K})$
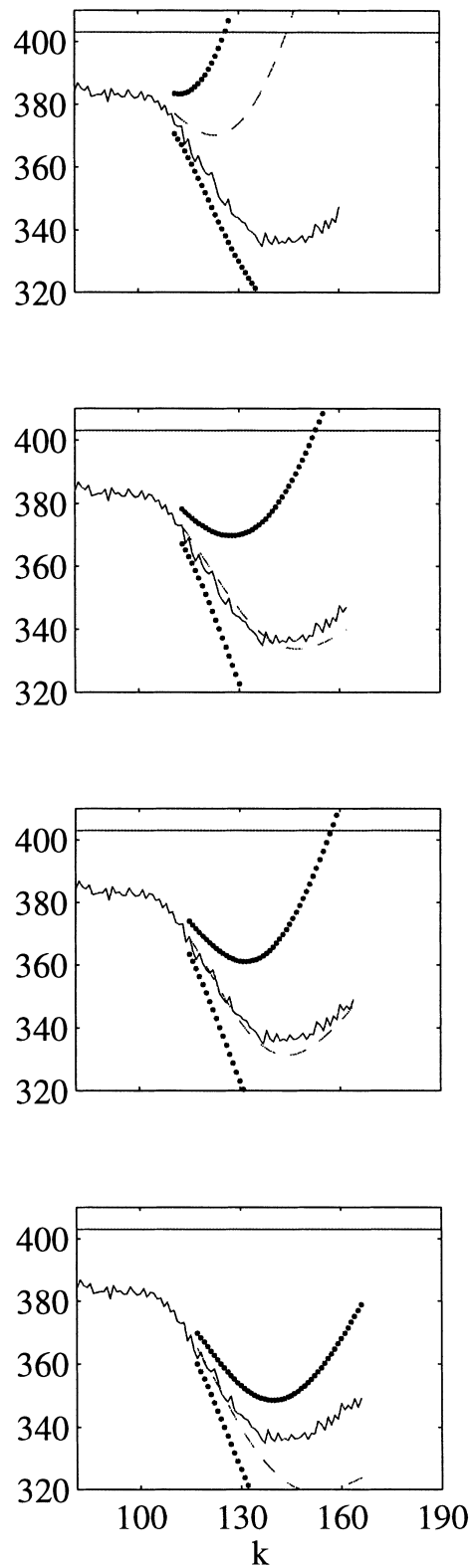

$\mathrm{T}^{2}\left(\mathrm{k}^{*}, \mathrm{k}\right)$
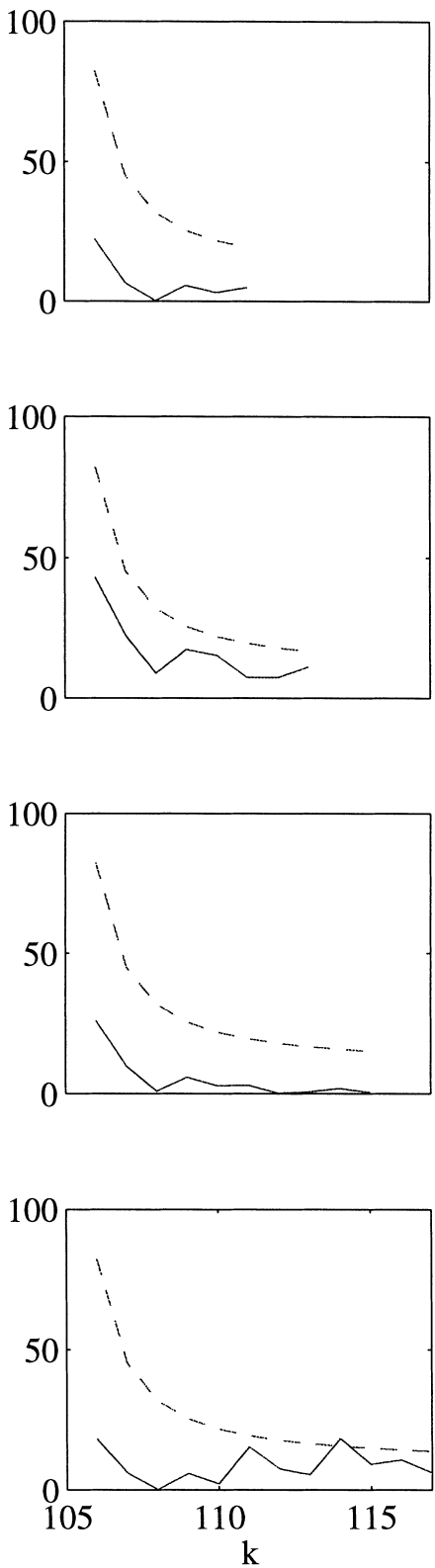

Fig. 8. $-0.375 \mathrm{~mol} / 1$ step in $C_{\mathrm{F}}$ and heat exchanger fouling occurs: (a) $k^{*}=111$, (b) $k^{*}=113$, (c) $k^{*}=115$ (d) $k^{*}=117$. 
Of course, using an incorrect process model does not always simply result in a smaller warning period - parametric errors can cause the model to incorrectly forecast future behavior. But for these situations, large forecast errors will result in large values of the $T^{2}$ statistic.

Nonstationary disturbances (e.g. a random walk) present a fundamental limitation for predicting future outputs accurately. The results in Fig. 9 demonstrate the difficulty of predicting when nonstationary or "unit root" disturbances occur, the bane of financial market analysts [23]. A random walk signal for $C_{\mathrm{F}}$ is created by,

$C_{\mathrm{F}}(k)=\frac{q}{q-1} a(k)$

where $a$ is white noise with variance, $\sigma_{a}^{2}=0.001$. Again, a ramp pseudo-disturbance is assumed. At $k=140$, both confidence limits for the temperature forecasts exceed the emergency limit, although the actual values do nota false alarm. However, the calculated $T^{2}$ statistic in Fig. 9 violates the confidence limit from (20) repeatedly; consequently, the future predictions should be ignored because the model has not predicted the future behavior accurately in the past. This result suggests that at least one of the necessary assumptions is not valid.

The previous CSTR simulations have used the linearized model to both generate the process data and to forecast future outputs. In general, it is not possible to accurately predict nonlinear process behavior over a wide range of conditions with a linearized model. But as long as the process can be adequately described by the linearized model, the predictions should still remain reasonably accurate. For a step change of $60 \mathrm{~K}$ in $T_{\mathrm{F}}$ during
$\mathrm{C}_{\mathrm{A}}(\mathrm{mol} / \mathrm{l})$
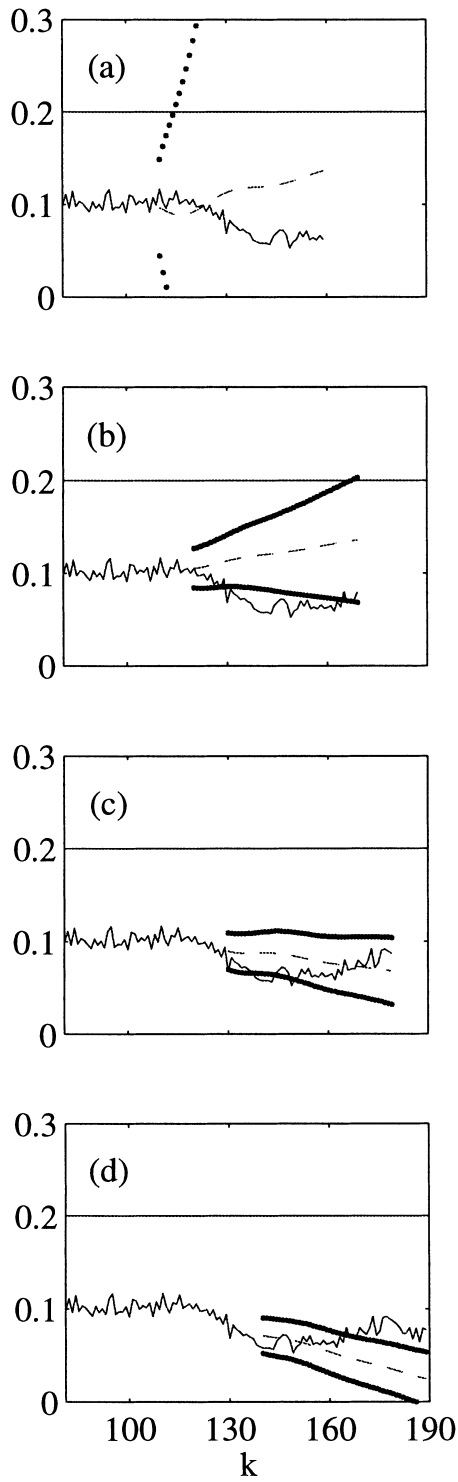

$\mathrm{T}(\mathrm{K})$
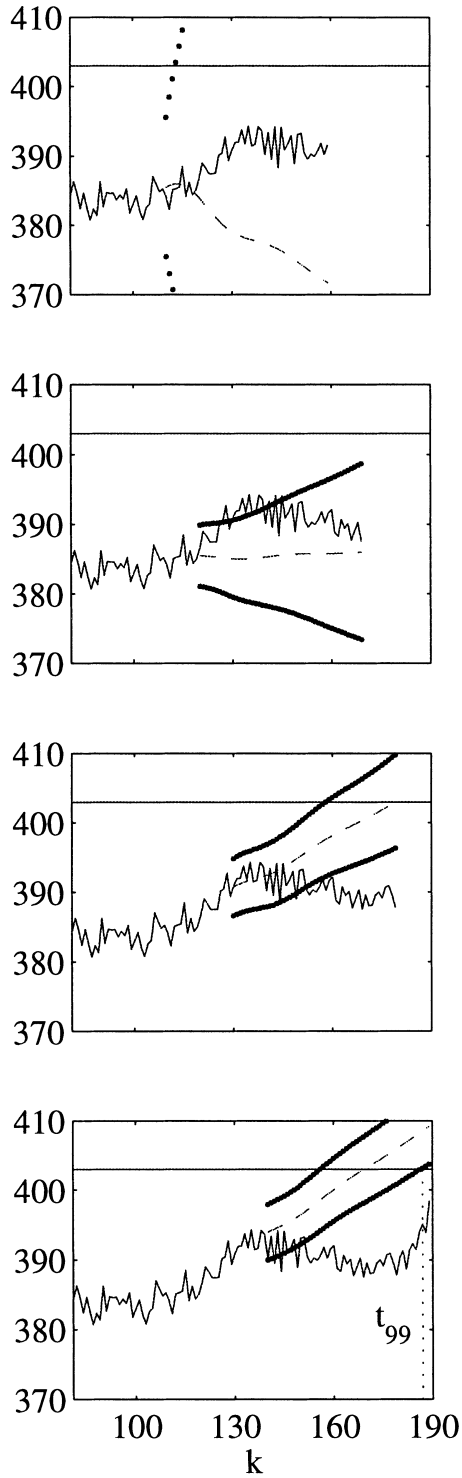

$\mathrm{T}^{2}\left(\mathrm{k}^{*}, \mathrm{k}\right)$
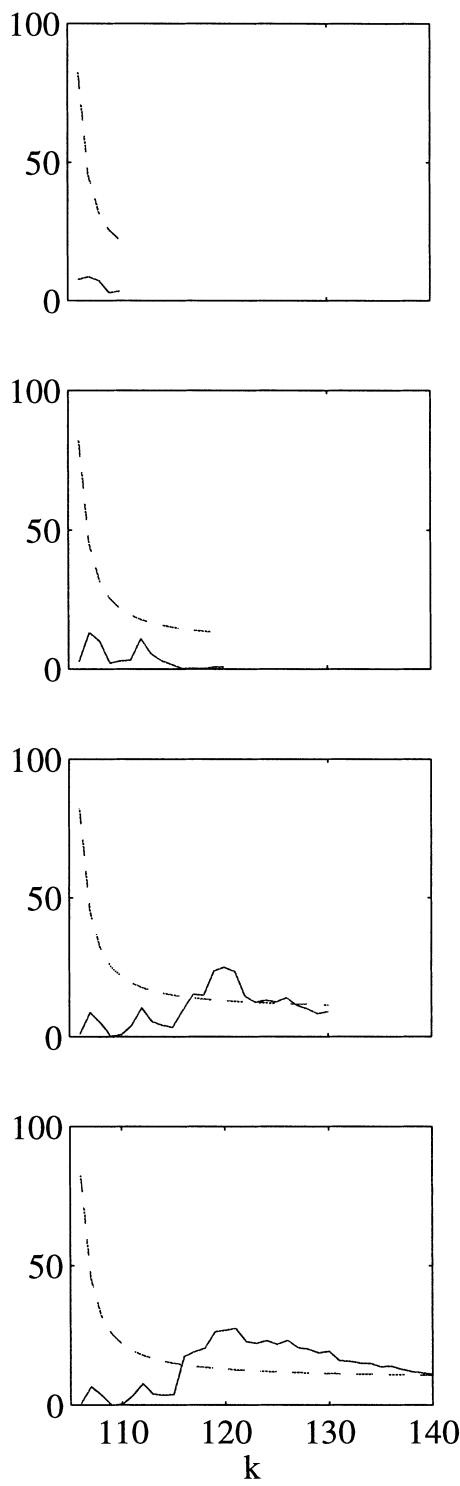

Fig. 9. A random walk disturbance in $C_{\mathrm{F}}$ : (a) $k^{*}=110$, (b) $k^{*}=120$, (c) $k^{*}=130$, (d) $k^{*}=140$. 
open-loop operation, the nonlinear process response cannot be predicted accurately by the linearized model, as shown in Fig. 10. In this case the predictions fail to predict the violation for $T$; they also incorrectly indicate that the temperature will return below the emergency limit and that $C_{\mathrm{A}}$ will violate its upper limit. However, the predictions are not reliable, as indicated by the multiple violations of the $T^{2}$ confidence limits for $k \geqslant 117$. In Fig. 11 the linear model is able to provide accurate predictions of the nonlinear process for a $60 \mathrm{~K}$ step disturbance in $T_{\mathrm{F}}$ during closed-loop operation. The temperature violation at $k=172$ is predicted at $k^{*}=135$, and $\Delta t_{\mathrm{w}}=37$.
Changing the prediction horizon, $N_{p}$, does not affect the predicted values, but can affect $\Delta t_{\mathrm{w}}$. Fig. 12 shows the effect of $N_{p}$ on predictions for the $-0.375 \mathrm{~mol} / 1 \mathrm{step}$ in $C_{\mathrm{F}}$ used in Fig. 6. For $N_{p}=10$, the violation is not detected because the prediction horizon does not include the time that the emergency limit is violated. For $N_{p} \geqslant 30$ the violation is detected, and the predictions for larger $N_{p}$ values are simply concatenated to the earlier predictions. The choice of $N_{p}$ essentially specifies the duration of the disturbance, because the predictions from (16) assume that the disturbance remains constant from $k$ to $k+N_{p}$. The value of $N_{p}$ should therefore be chosen to give just enough time to take corrective
$\mathrm{C}_{\mathrm{A}}(\mathrm{mol} / \mathrm{l})$
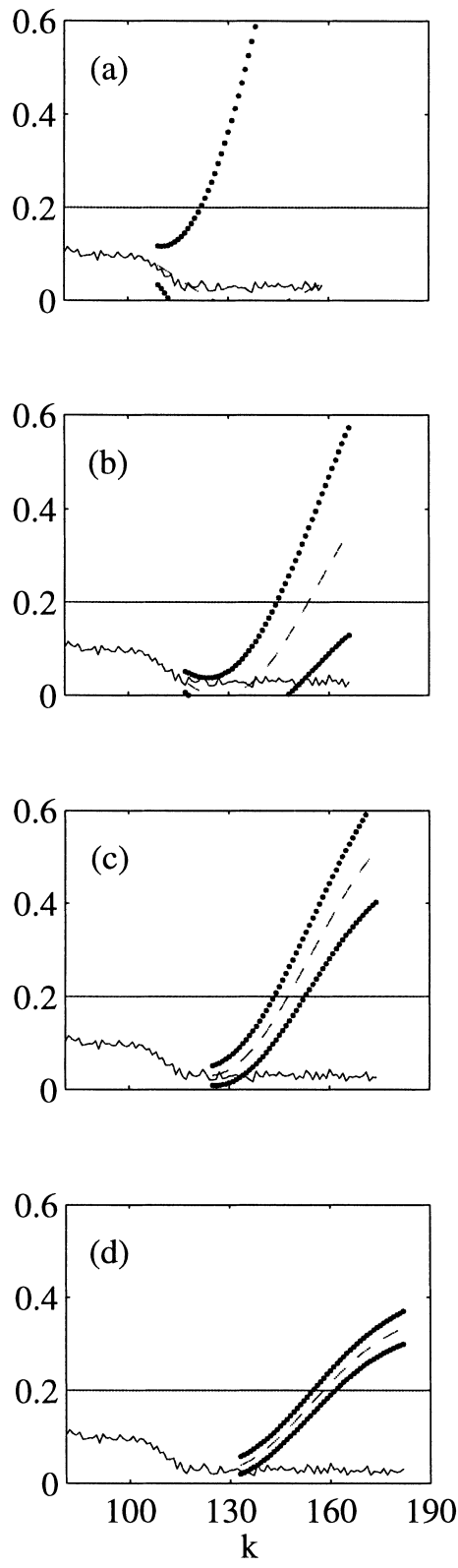

$\mathrm{T}(\mathrm{K})$
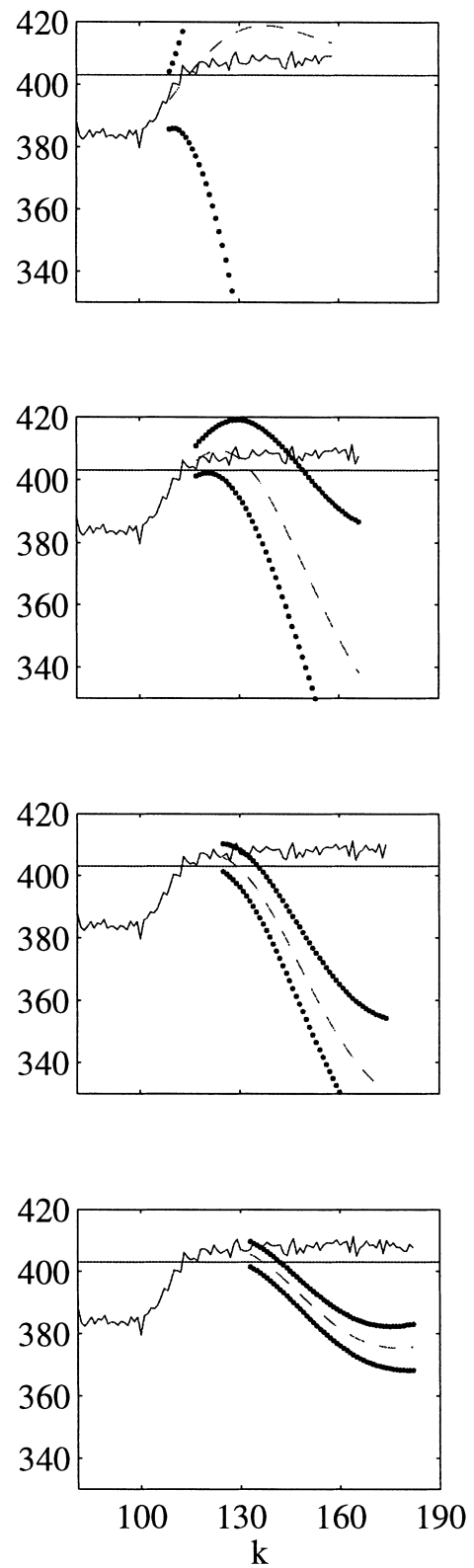

$\mathrm{T}^{2}\left(\mathrm{k}^{*}, \mathrm{k}\right)$
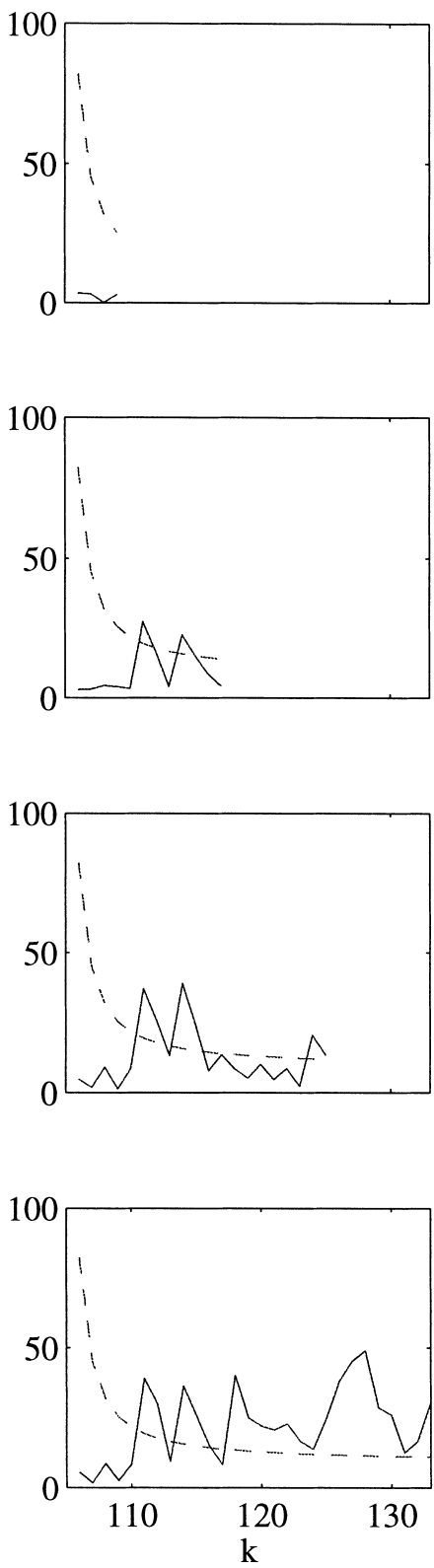

Fig. 10. Step increase in $T_{\mathrm{F}}$ for nonlinear system: (a) $k^{*}=110$, (b) $k^{*}=118$, (c) $k^{*}=126$, (d) $k^{*}=134$. 
action, because a larger value of $N_{p}$ is a bigger assumption about the disturbance.

Table 2 summarizes the results of the CSTR simulation study.

\section{Conclusions}

A novel process monitoring concept has been introduced based on using predictions of process variables to determine if an emergency limit will be violated in the future. The proposed approach complements existing fault diagnosis techniques. Normally after a fault is detected, a fault diagnosis begins. If diagnosis is not informative, e.g. the fault has not been seen before, predicting future outputs may help determine the impact of the fault, and provide insight to the corrective action that is needed.

Simulation results for linear models indicate that the proposed procedure for estimating the pseudo-disturbance and forecasting future outputs works well when the model and the noise statistics are known reasonably
$\mathrm{C}_{\mathrm{A}}(\mathrm{mol} / \mathrm{l})$
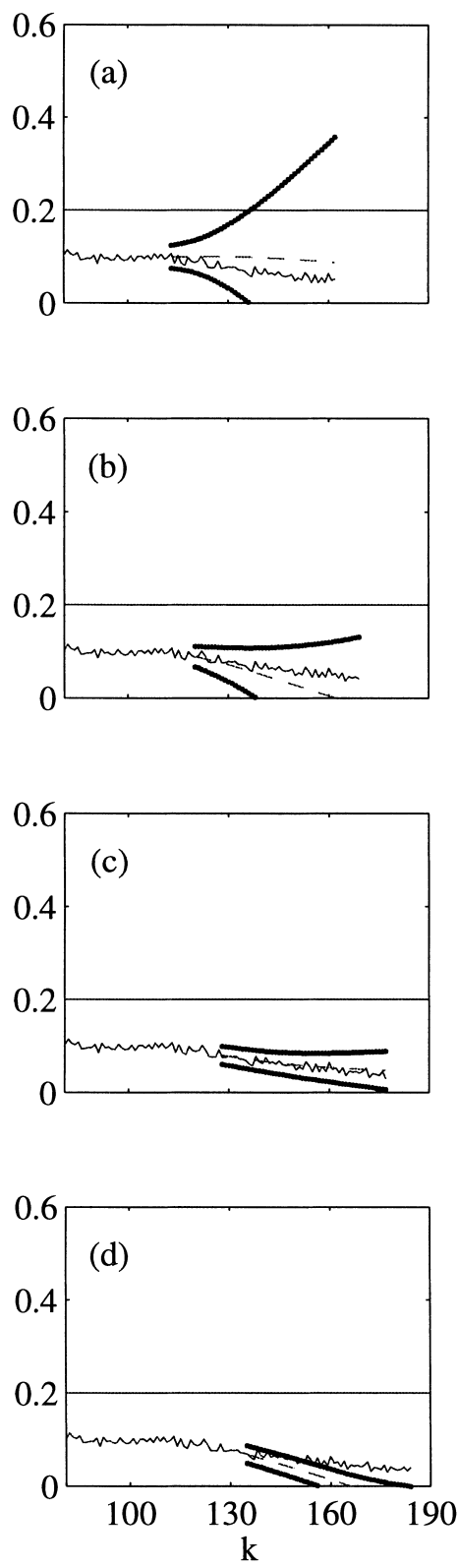

$\mathrm{T}(\mathrm{K})$
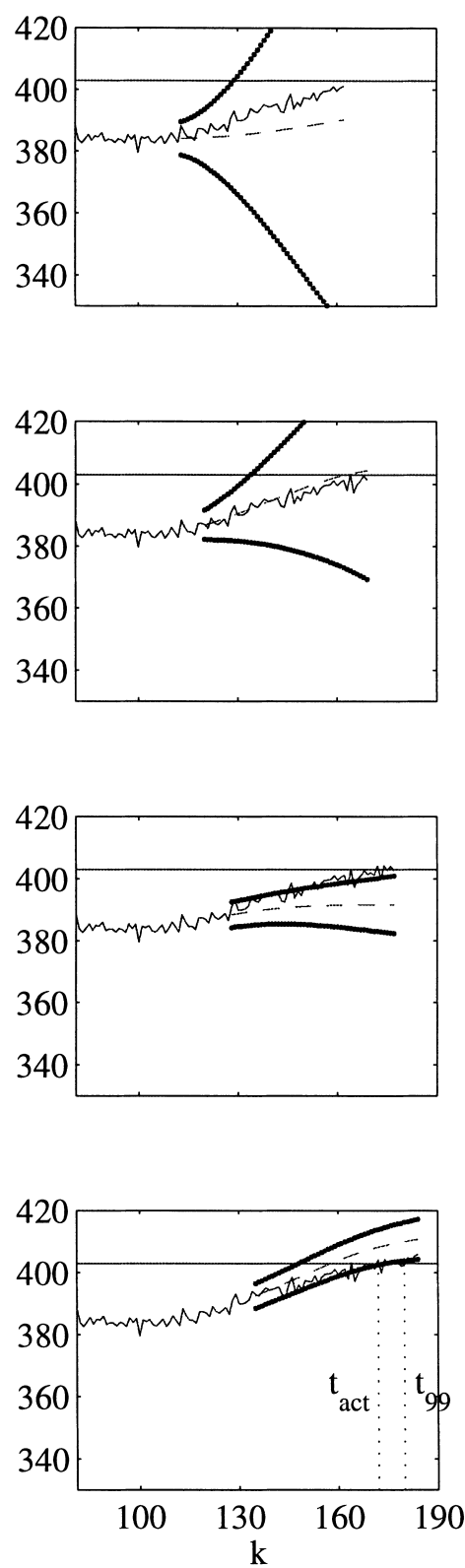

$\mathrm{T}^{2}\left(\mathrm{k}^{*}, \mathrm{k}\right)$
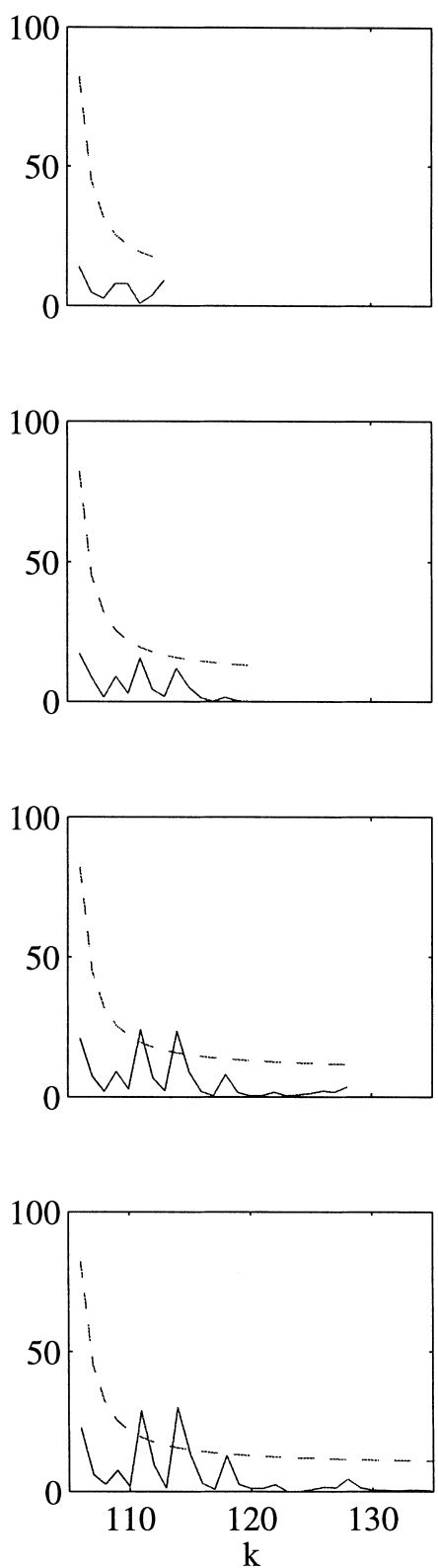

(d.) Predictions made at $\mathrm{k}^{*}=134$

Fig. 11. Step increase in $T_{\mathrm{F}}$ for closed-loop, nonlinear system: (a) $k^{*}=112$, (b) $k^{*}=119$, (c) $k^{*}=127$, (d) $k^{*}=134$. 
well, and a modest amount data are available for an accurate estimate of the pseudo-disturbance. Process nonlinearities, model mismatch, and nonstationary disturbances can have detrimental effects on future predictions. But inaccurate predictions can be readily identified because they lead to repeated violations of the proposed $T^{2}$ test. Using the $T^{2}$ statistic as a guide, one can choose to ignore the future predictions. Furthermore, use of the $T^{2}$ test reduces the number of false alarms, as shown in the random walk simulation.

In this paper, the proposed process monitoring strategy has been based on forecasts for linear state-space models. The general approach, however, can be used with other types of linear models (e.g. step response or transfer function models) or nonlinear models (e.g. physical or empirical models).

Forecasting future process measurements under abnormal conditions should be done with both caution and humility. Complex processes with time-varying disturbances probably cannot be predicted well with any reasonable mathematical model. However, it should be possible to use a method such as the one proposed in this research for processes that are locally linear and subject to sustained disturbances. Furthermore, any prediction methodology should use a metric like the $T^{2}$
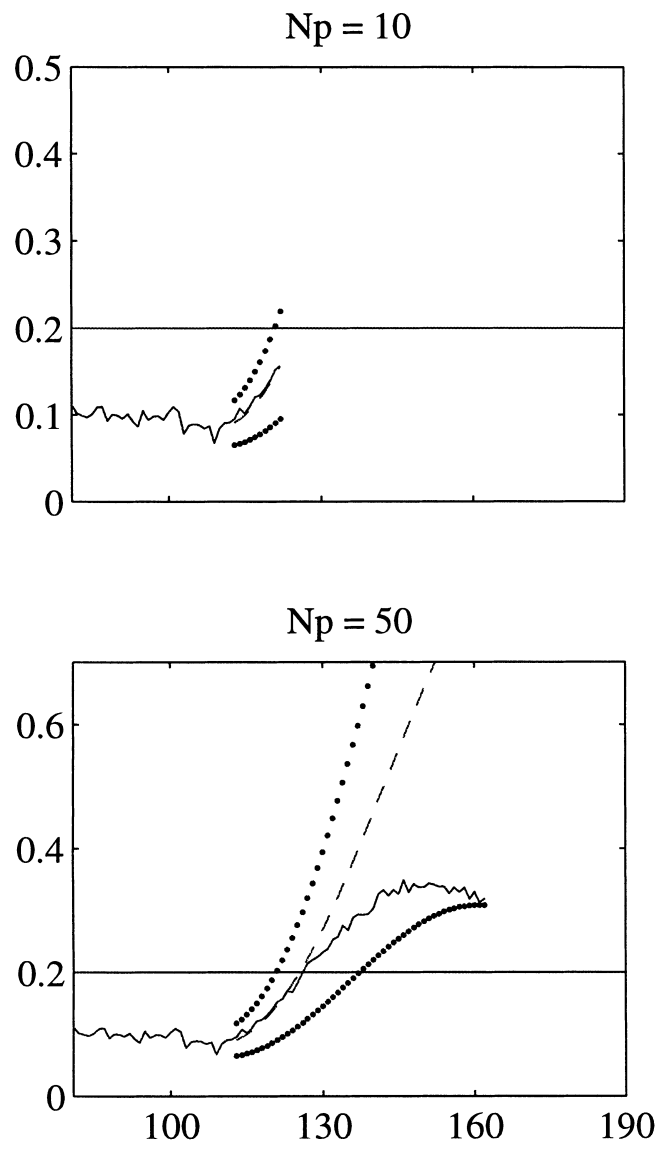

Table 2

CSTR simulation results

\begin{tabular}{lllll}
\hline Fig. & Disturbance & Actual & $\Gamma(k)$ & $\Delta t_{\mathrm{w}}$ \\
\hline 3 & $T_{\mathrm{F}}$ & Step & Step & - \\
4 & $C_{\mathrm{F}}$ & Step & Step & 22 \\
5 & $C_{\mathrm{F}}$ & Ramp & Ramp & 32 \\
6 & $C_{\mathrm{F}}$ & Step & Ramp & 17 \\
7 & $C_{\mathrm{F}}{ }^{\mathrm{a}}$ & Step & Ramp & 29 \\
8 & $C_{\mathrm{F}}{ }^{\mathrm{a}}{ }^{\mathrm{b}}$ & Step & Ramp & 11 \\
9 & $C_{\mathrm{F}}$ & Random walk & Ramp & - \\
10 & $T_{\mathrm{F}}{ }^{\mathrm{c}}$ & Step & Ramp & - \\
11 & $T_{\mathrm{F}}{ }^{\mathrm{a}}{ }^{\mathrm{c}}$ & Step & Ramp & 37 \\
\hline
\end{tabular}

a Closed-loop operation.

b Plant-model mismatch.

c Nonlinear CSTR equation used to generate data.

statistic as a tool for assessing whether the future predictions are reliable, based on the performance of past predictions.

\section{Appendix. Estimation $\delta$}

The following derivation follows Section 7.2 in [8]. The state space model of the normal system is given by,
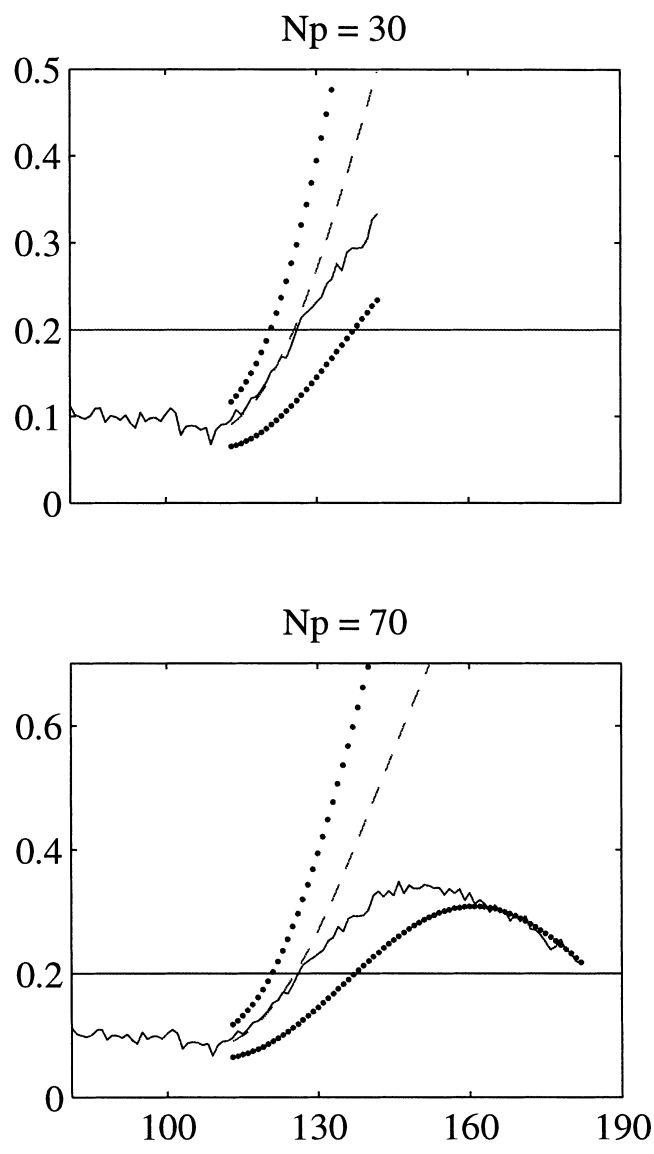

Fig. 12. Effect of prediction horizon, $N_{p}$, on $C_{\mathrm{A}}$ predictions. 


$$
\begin{aligned}
x^{0}(k+1) & =A x^{0}(k)+B u(k)+w(k) y(k) \\
& =C x^{0}(k)+v(k)
\end{aligned}
$$

If the true system in (1) is written in terms of the pseudo-disturbance, $\delta\left(k, k_{0}\right)$,

$$
\begin{aligned}
x(k+1) & =A x(k)+B u(k)+\delta\left(k, k_{0}\right)+w(k) y(k) \\
& =C x(k)+v(k)
\end{aligned}
$$

Define the quantities $\alpha\left(k, k_{0}\right), \quad \beta\left(k, k_{0}\right), \rho\left(k, k_{0}\right)$ to describe the fault signature on the true state, the estimated state, and the Kalman filter residuals,

$$
\begin{aligned}
& \alpha\left(k, k_{0}\right) \triangleq x(k)-x^{0}(k) \\
& \beta\left(k, k_{0}\right) \triangleq \hat{x}(k)-\hat{x}^{0}(k) \\
& \rho\left(k, k_{0}\right) \triangleq \epsilon(k)-\epsilon^{0}(k)
\end{aligned}
$$

where the superscript ${ }^{0}$ denotes the quantities associated with the model and Kalman filter for the unperturbed system. The residuals $\epsilon^{0}$ are distributed as $N(0, \bar{\Sigma})$. Without loss of generality, assume that $\bar{\Sigma}=\mathrm{I}$. Using (A-1) and (A-2), the following recursions can be derived,

$$
\begin{aligned}
\alpha\left(k, k_{0}\right) & =A \alpha\left(k-1, k_{0}\right)+\delta(k-1) \\
\beta\left(k, k_{0}\right) & =A \beta\left(k-1, k_{0}\right)+K \rho\left(k, k_{0}\right) \\
\rho\left(k, k_{0}\right) & =C\left[\alpha\left(k, k_{0}\right)-A \beta\left(k-1, k_{0}\right)\right]
\end{aligned}
$$

For the steady state Kalman filter, (A-4) can be written as,

$\rho\left(k, k_{0}\right)=\sum_{i=k_{0}}^{k-1} C \bar{A}^{k-i-1} \delta(i)$

Once the disturbance form is specified, $\delta(k)=\Gamma(k) \delta$ can be substituted into (A-5),

$$
\begin{aligned}
& \rho\left(k, k_{0}\right)=\left(\sum_{i=k_{0}}^{k-1} C \bar{A}^{k-i-1} \Gamma(i)\right) \delta \\
& \rho\left(k, k_{0}\right)=\tilde{\rho}\left(k, k_{0}\right) \delta
\end{aligned}
$$

From the definition of $\rho$,

$$
\epsilon(k)=\tilde{\rho}\left(k, k_{0}\right) \delta+\epsilon^{0}(k)
$$

Recall that $\epsilon(k)$ are the measured residuals from the Kalman filter for the true system, and $\tilde{\rho}\left(k, k_{0}\right)$ are precomputable using the system matrices and knowledge of the disturbance form. For $N$ measurements of $\epsilon(k)$ (let $\left.k_{0}=0\right)$,

$$
\left[\begin{array}{c}
\epsilon(1) \\
\vdots \\
\epsilon(N)
\end{array}\right]=\left[\begin{array}{c}
\tilde{\rho}(1) \\
\vdots \\
\tilde{\rho}(N)
\end{array}\right] \delta+\left[\begin{array}{c}
\epsilon^{0}(1) \\
\vdots \\
\epsilon^{0}(N)
\end{array}\right]
$$

Because the random errors, $\epsilon^{0}$, are uncorrelated, the least squares estimate of $\delta$ is given by,

$$
\begin{aligned}
& \hat{\delta}=\left(\left[\tilde{\rho}^{T}(1) \cdots \tilde{\rho}^{T}(N)\right]\left[\begin{array}{c}
\tilde{\rho}(1) \\
\vdots \\
\tilde{\rho}(N)
\end{array}\right]\right)^{-1} \\
& \left(\left[\tilde{\rho}^{T}(1) \cdots \tilde{\rho}^{T}(N)\right]\left[\begin{array}{c}
\epsilon(1) \\
\vdots \\
\epsilon(N)
\end{array}\right]\right)
\end{aligned}
$$

The solution (scaled by $\bar{\Sigma}^{-1}$ for $\bar{\Sigma} \neq I$ ) can be compactly written as (11).

\section{References}

[1] I. Nimmo, Adequately address abnormal operations, Chem. Eng. Progress 91 (9) (1995) 36-45.

[2] G. Box, A. Luceño, Statistical Control by Monitoring and Feedback, John Wiley, NY, 1997.

[3] P. Frank, Fault diagnosis in dynamic systems using analytical and knowledge-based redundacy, Automatica 26 (1990) 459-474.

[4] R. Isermann, Integration of fault detection and diagnosis methods, in: Proceedings of the IFAC Fault Detection, Supervision and Safety for Technical Processes, Espoo, Finland, 1994.

[5] J. Davis, B. Bakshi, K. Kosanovich, M. Piovoso, Process Data Analysis and Interpretation, in: J. Wei, K.B. Bischoff, M.M. Denn (Eds), Vol. 25 of Advances in Chem. Eng, Academic Press, New York, 2000.

[6] M. Kramer, R. Mah, Model-based monitoring, in: Proceedings of the Second International Conference on Foundations of Computer Aided Process Operations, Austin, TX, 1994, pp. 45-71.

[7] G. Stephanopoulos, C. Han, Intelligent systems in process engineering: a review, in: Preprints of the Fifth Int. Symposium on Process Systems Eng., Kyongju, Korea, 1994, pp. 1339-1366.

[8] M. Basseville, I. Nikiforov, Detection of Abrupt Changes, Prentice-Hall, Englewood Cliffs, NJ, 1995.

[9] J. Gertler, Fault Detection and Diagnosis in Engineering Systems, Marcel Dekker, NY, 1998

[10] R. Patton, P. Frank, R. Clark, Fault Diagnosis in Dynamic Systems: Theory and Application, Prentice-Hall, Hertfordshire, 1989.

[11] A. Willsky, A survey of design methods for failure detection in dynamic systems, Automatica 12 (1976) 601-611.

[12] J. Chen, R. Patton, Optimal filtering and robust fault diagnosis of stochastic systems with unknown disturbances, IEE Proc.Control Theory Appl. 43 (1996) 31-36.

[13] P. Frank, X. Ding, Survey of robust residual generation and evaluation methods in observer-based fault detection systems, J. Process Control 7 (1997) 403-424.

[14] R. Patton, Robust model-based fault diagnosis: the state of the art, in: Proceedings of the IFAC Fault Detection, Supervision and Safety for Technical Processes, Espoo, Finland, 1994, pp. 1-23.

[15] B. Juricek, W. Larimore, D. Seborg, Early detection of alarm situations using model predictions, in: Proc. IFAC Workshop on On-Line Fault Detection, Lyon, France, 1998.

[16] P. Nomikos, J. MacGregor, Monitoring of batch processes using multi-way PCA, AIChE J. 40 (1994) 1361-1375.

[17] G. Chen, T. McAvoy, Predictive on-line monitoring of continuous processes, J. Process Control 8 (1998) 409-420. 
[18] D. Ruppen, D. Bonvin, D. Rippin, Implementation of adaptive optimal operation for a semi-batch reaction system, Comp. \& Chem. Eng. 22 (1997) 185-199.

[19] N. Ricker, Model predictive control with state estimation, Ind. Eng. Chem. Res. 29 (1990) 374-382.

[20] S. Qin, T. Badgwell, An overview of industrial model predictive control technology, in: AIChE Symposium Series 316: CPC-V, Lake Tahoe, CA, 1996, pp. 232-256.

[21] G. Goodwin, K. Sin, Adaptive Filtering, Prediction and Control, Prentice-Hall, Englewood Cliffs, NJ, 1984.

[22] A. Gelb, Applied Optimal Estimation, M.I.T. Press, Cambridge, MA, 1974

[23] H. Lütkepohl, Introduction to Multiple Time Series Analysis, Springer-Verlag, Berlin, 1991
[24] B. Friedland, Treatment of bias in recursive filtering, IEEE Trans. Automatic Control AC-14 (1969) 359-367.

[25] M. Darouach, M. Zasadzinski, Unbiased minimum variance estimation for systems with unknown exogenous inputs, Automatica 33 (1997) 717-719.

[26] D. Haessig, B. Friedland, Separate-bias estimation with reducedorder Kalman filters, IEEE Trans. Automatic Control 43 (1998) 983-987.

[27] T. Anderson, An Introduction to Multivariate Statistical Analysis, John Wiley, NY, 1984.

[28] M. Basseville, Information criteria for residual generation and fault detection and isolation, Automatica 33 (1997) 783-803.

[29] D. Seborg, T. Edgar, D. Mellichamp, Process Dynamics and Control, John Wiley, NY, 1989. 